\title{
Multipartite dark matter with scalars, fermions and signatures at LHC
}

\author{
Subhaditya Bhattacharya, ${ }^{a}$ Purusottam Ghosh ${ }^{a}$ and Narendra Sahu ${ }^{b}$ \\ ${ }^{a}$ Department of Physics, Indian Institute of Technology Guwahati, \\ North Guwahati, Assam 781039, India \\ ${ }^{b}$ Department of Physics, Indian Institute of Technology Hyderabad, \\ Kandi, Sangareddy, Telangana 502285, India \\ E-mail: subhab@iitg.ac.in, pghoshiitg@gamil.com, nsahu@iith.ac.in
}

ABSTRACT: Basic idea of this analysis is to achieve a two-component dark matter (DM) framework composed of a scalar and a fermion, with non-negligible DM-DM interaction contributing to thermal freeze out (hence relic density), but hiding them from direct detection bounds. We therefore augment the Standard Model (SM) with a scalar singlet $(S)$ and three vectorlike fermions: two singlets $\left(\chi_{1}, \chi_{2}\right)$ and a doublet $(N)$. Stability of the two DM components is achieved by a discrete $\mathcal{Z}_{2} \times \mathcal{Z}_{2}^{\prime}$ symmetry, under which the additional fields transform suitably. Fermion fields having same $\mathcal{Z}_{2} \times \mathcal{Z}^{\prime}{ }_{2}$ charge $\left(N, \chi_{1}\right.$ in the model) mix after electroweak symmetry breaking (EWSB) and the lightest component becomes one of the DM candidates, while scalar singlet $S$ is the other DM component connected to visible sector by Higgs portal coupling. The heavy fermion $\left(\chi_{2}\right)$ plays the role of mediator to connect the two DM candidates through Yukawa interaction. This opens up a large parameter space for the heavier DM component through DM-DM conversion. Hadronically quiet dilepton signature, arising from the fermion dark sector, can be observed at Large Hadron Collider (LHC) aided by the presence of a lighter scalar DM component, satisfying relic density and direct search bounds through DM-DM conversion.

Keywords: Beyond Standard Model, Cosmology of Theories beyond the SM

ARXIV EPRINT: 1809.07474 


\section{Contents}

1 Introduction 1

2 The model 3

$\begin{array}{lll}2.1 & \text { Constraints on the model parameters } & 7\end{array}$

2.2 Possible multipartite DM scenarios 8

3 Review of single component DM frameworks with $N_{1}$ and $S \quad 9$

3.1 Single component fermion DM $\left(N_{1}\right) \quad 9$

$\begin{array}{ll}3.2 \text { Single component scalar DM }(S) & 10\end{array}$

4 Two component DM with $N_{1}$ and $S \quad 12$

$\begin{array}{lll}4.1 \text { Coupled Boltzmann equations } & 12\end{array}$

$\begin{array}{lll}4.2 & \text { Relic density and direct search outcome } & 14\end{array}$

5 Two component DM in presence of additional heavy scalar 23

6 Collider searches at LHC 25

7 Possible implications to inflation and reheating 33

$\begin{array}{lll}8 & \text { Summary } & 35\end{array}$

A Single component vector-like fermion DM 36

$\begin{array}{ll}\text { B Higgs invisible decay constraint } & 38\end{array}$

C Invisible decay constraint of $Z \quad 40$

\section{Introduction}

Observation of galactic rotation curves [1, 2], gravitational lensing and anisotropies in cosmic microwave background [3] collectively hint towards the existence of a cosmologically stable dark matter (DM) component in the present Universe [4]. However, there is no such particle candidate exist within the standard model (SM), which can behave as DM. Hence physics beyond the SM is inevitable. Hitherto the only information known about DM is its relic abundance and is precisely determined by Wilkinson Microwave Anisotropy Probe (WMAP) [5] and PLANCK [6] to be $\Omega_{\mathrm{DM}} h^{2}=0.1161 \pm 0.0028$. Apart from this, we don't have any other information about DM, such as its mass, spin, interaction etc. As a result, the nature of DM being a scalar, a fermion, or a vector boson or an admixture of them can not be avoided. In addition to gravity, if the DM is weakly interacting to visible sector, then it can thermalise in the early Universe at a temperature above its mass scale. As the Universe cools down due to Hubble expansion, the DM freezes-out from the thermal plasma at a temperature below its mass scale and gets redshifted since then. It 
is miraculous that the observed DM abundance implies to thermal freeze-out cross-section of DM: $\langle\sigma|v|\rangle \approx 10^{-36} \mathrm{~cm}^{2}$, of typical weak interaction strength and therefore it is largely believed that the DM is a weakly interacting massive particle (WIMP) [7].

However, the WIMP paradigm suffers from a serious threat due to the non-observation of DM in direct search experiments. In fact, in a few years from now the DM-nucleon cross-section measured at direct search experiments may hit the neutrino floor [8], where neutrino-nucleon cross-section will be a huge background for DM detection. The main problem in a WIMP paradigm is that the interactions which lead to the freeze-out of DM in the early Universe, also yields DM-nucleon cross-section in direct search experiments in the present epoch, such as LUX [9], XENON [10, 11], PANDA [12] etc. The same is true for non-observation of DM in collider searches as well. The only difference for a WIMP of $\sim 100 \mathrm{GeV}$ is that the production of DM at collider is suppressed (with no electromagnetic or strong interactions with $\mathrm{SM}$ ), so that non-observation of DM in collider provides less constraint than those of direct searches at terrestrial laboratories.

Multipartite DM frameworks [13-30] can provide a cushion to the tension of WIMP like particles to satisfy simultaneously relic density and direct search constraints. This is essentially due to some processes which can still contribute to the depletion of DM number density for thermal freeze-out, but do not contribute to direct search cross-sections. The main two contributions of such kind can arise from: (i) Co-annihilation of DM with a heavier particle, which can not be produced in direct search for kinematic suppression [21, 31] or (ii) DM-DM interactions, where the heavier DM component can annihilate to the lighter one to yield thermal relic, but do not contribute to direct searches of DM [20,21].

Our paper investigates one of the simplest of such cases, where we assume the presence of two DM components: one scalar $(S)$ and a fermion $\left(N_{1}\right)$. While both DMs have been studied as individual components [21,32-34], we study the interplay of DM-DM interactions when they are present together. In order to enhance such interactions, we insert an additional singlet fermion field $\left(\chi_{2}\right)$, which works as a mediator and carries the interaction through a Yukawa term. We thereafter demonstrate that a large parameter space becomes available to each DM components, whichever is heavy, saved from direct search bound thanks to enhanced DM-DM interactions. The lighter DM component however, has the fate similar to that of a single component case, particularly when direct search is concerned. This shows that scalar DM can only be present in the vicinity of Higgs resonance $\left(m_{S} \sim m_{h} / 2\right)$ when it is lighter than fermion DM. The presence of an additional heavy scalar $\left(S_{H}\right)$ in the model can however yield a larger parameter space for the scalar DM (even when it is lighter than fermion DM). Efforts have already been made to accommodate scalar and fermion DM together in a single framework [14, 17], but most often the role of DM-DM interactions has been subdued and the outcome is predictive and severely constrained.

Collider signatures of both the DM components have also been addressed before (see for example, $[35,36])$. Unfortunately, it turns out that neither the scalar nor the fermion DM (in their single component realisation) has a possibility of producing signal excess over SM background in near future run of Large Hadron Collider (LHC), ${ }^{1}$ while satisfying

\footnotetext{
${ }^{1}$ Fermion DM with singlet-doublet mixing may however yield a displaced vertex signature [35].
} 
relic density and direct search constraints. We however demonstrate here, the presence of a lighter scalar DM component helps in identifying hadronically quiet dilepton signal (a characteristic signature for the charged lepton components present in the fermion dark sector) at LHC, which was otherwise impossible due to unsurpassable SM background contribution. This is accessible due to the freedom of utilising a larger missing energy cut, resulting from a larger allowed mass difference between the fermion DM and its charge companions, thanks to the presence of a lighter DM component and non-negligible DM-DM interactions in the set up to satisfy relic density and direct search bounds.

The paper is organised as follows. We first introduce the model framework (in section 2). After reviewing relic density and direct search constraints on the individual DM components for single component frameworks (in section 3), we discuss in details the case of two-component set up poised with DM-DM conversion (in section 4). We also point out to the possibilities of having an additional heavy scalar in the framework (in section 5). We then elucidate signatures of fermion dark sector at LHC accessible through two component set up (in section 6). We also briefly discuss possible cosmological effect on DM particles due to early universe inflation and reheating (in section 7 ). Finally we summarise and conclude (in section 8). Some illustrative features of fermion DM, Higgs invisible decay and $Z$ invisible decay constraints on the model are detailed in appendices A, B and C respectively.

\section{The model}

The model addressed here, accommodates two single component DM frameworks together: (i) a real scalar singlet DM $(S)$, connected to SM through Higgs portal $[21,32,33,37]$ and $(i i)$ a fermion DM arising out of the admixture of vectorlike fermion (VF) doublet, $N=\left(\begin{array}{ll}N^{0} & N^{-}\end{array}\right)^{T}$ and a vectorlike fermion singlet $\chi_{1}[34,35,38]$, where lightest component becomes a DM. Stability of a single DM can be ensured by an additional discrete $\mathcal{Z}_{2}$ symmetry, under which the DM transforms nontrivially. However, when two DMs are present together, the stability of both components can be ensured by enhancing the symmetry to $\mathcal{Z}_{2} \times \mathcal{Z}_{2}^{\prime}$, where two DMs transform differently under the symmetry as we will illustrate shortly. Two-component DM frameworks are naturally disfavoured from direct search as each DM component acquires smaller relic density resulting enhanced annihilation cross-section to SM for freeze out. This enhances direct search cross-sections for both the DM components (resulting from same interaction vertices). This is the reason that most of the existing scalar-fermion DM scenarios have been severely discarded by stringent direct search limits $[14,17]$. However, DM-DM interactions may come to rescue as the freeze-out of the heavier component will then be additionally driven by its annihilation to lighter DM component, which do not contribute to direct search cross-section of that component. In order to enhance such interplay, we have introduced an additional vectorlike singlet fermion $\chi_{2}$, which behaves like a messenger between the two DM components. The interaction between the two DM components and their individual connection to the visible sector $(\mathrm{SM})$ are shown by a schematic diagram in figure 1 . Under the $\mathcal{Z}_{2} \times \mathcal{Z}_{2}^{\prime}$ symmetry, additional dark fields transform as: $N[-,+], \chi_{1}[-,+], \chi_{2}[+,-]$ and $S[-,-]$, where all SM fields remain invariant: SM $[+,+]$. The quantum numbers under the SM gauge group 


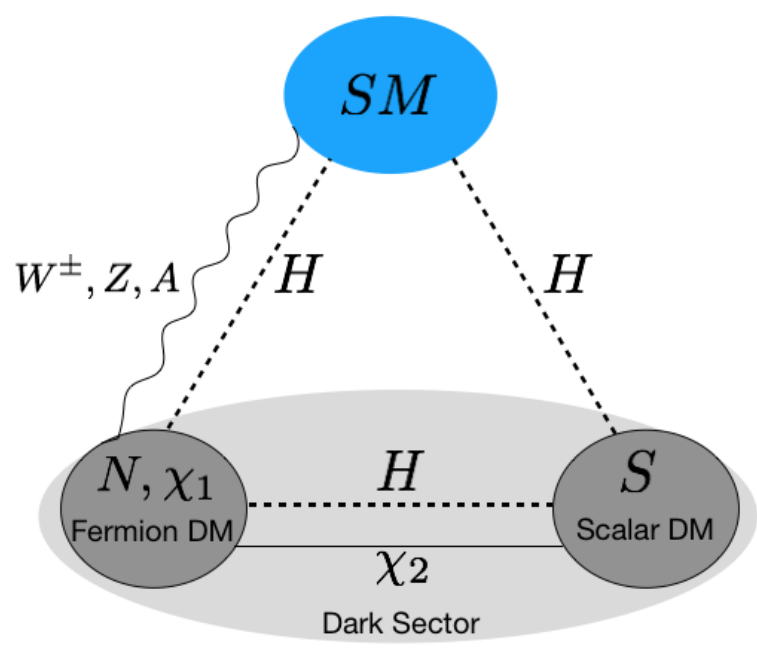

Figure 1. Schematic diagram showing the interactions between scalar and fermion DM components and that to SM particles.

\begin{tabular}{|c|ccccc|}
\hline Dark Fields & \multicolumn{4}{|c|}{$\underbrace{\mathrm{SU}(3)_{C} \times \mathrm{SU}(2)_{L} \times \mathrm{U}(1)_{Y}} \times \mathcal{Z}_{2} \times \mathcal{Z}^{\prime}{ }_{2}$} \\
\hline$N=\left(\begin{array}{l}N^{0} \\
N^{-}\end{array}\right)$ & 1 & 2 & -1 & - & + \\
\hline$\chi_{1}$ & 1 & 1 & 0 & - & + \\
\hline$\chi_{2}$ & 1 & 1 & 0 & + & - \\
\hline$S$ & 1 & 1 & 0 & - & - \\
\hline
\end{tabular}

Table 1. Dark sector fields and their corresponding quantum numbers under $\mathcal{G} \equiv \mathrm{SU}(3)_{C} \times$ $\mathrm{SU}(2)_{L} \times \mathrm{U}(1)_{Y} \times \mathcal{Z}_{2} \times \mathcal{Z}_{2}{ }_{2}$.

$\mathrm{SU}(3)_{C} \times \mathrm{SU}(2)_{L} \times \mathrm{U}(1)_{Y}$ and $\mathcal{Z}_{2} \times \mathcal{Z}_{2}^{\prime}$ symmetry for these additional fields are shown in the table 1. It is remarkable that these additional fermions $\chi_{1}, \chi_{2}$ and $N$ are verctorlike and hence they don't introduce any extra anomalies. This is easy to see through the chiral gauge anomaly free condition coming from the one loop triple gauge boson vertex, which reads [39]:

$$
\sum_{\text {rep }} \operatorname{Tr}\left[\left\{T_{L}^{a}, T_{L}^{b}\right\} T_{L}^{c}\right]-\operatorname{Tr}\left[\left\{T_{R}^{a}, T_{R}^{b}\right\} T_{R}^{c}\right]=0
$$

Here, $T$ denotes the generators for the SM gauge group and $L, R$ denotes the interactions of left or right chiral fermions with the gauge bosons. It is straightforward to see, that while the SM satisfies the anomaly free condition because of the presence of a quark family to each lepton family [39, 40], the additional vector like fermions introduced here, have the left chiral components transforming similarly to the right chiral ones under the SM gauge symmetry. Therefore, the model is anomaly free.

In table 1 , we note that $N$ and $\chi_{1}$ have similar $\mathcal{Z}_{2} \times \mathcal{Z}^{\prime}{ }_{2}$ charges. Hence they mix with each other after the SM Higgs acquires a vacuum expectation value (vev), while the other 
fermion $\chi_{2}$ does not. The lightest of such singlet-doublet admixture can be one fermion DM component of this model. The scalar singlet $S$ also have different charge assignment from that of all the other fermion fields, allowing it to be stabilized to form another DM component. The key feature of this model is the possibility of writing a Yukawa interaction between $\chi_{1}, \chi_{2}$, and $S$ by the assigned $\mathcal{Z}_{2} \times \mathcal{Z}_{2}^{\prime}$ charges, which adds to the possible DMDM interactions as we explain below. This particular feature segregates this model from earlier attempts of two component scalar-fermion DM set-up like in $[14,17]$, where DMDM interactions were small, so the model becomes strongly constrained by direct search or from the case where fermion DM doesn't have an interaction with visible sector (excepting at the loop level) to credit a large share of relic density to it and thus constraining the model to a particular possibility.

Let us now describe the Lagrangian for the model, which can be segregated into three parts, constituting the vector like fermion sector, scalar sector and the interaction between the fermion and scalar sector as follows:

$$
\mathcal{L} \supset \mathcal{L}^{\mathrm{VF}}+\mathcal{L}^{\text {Scalar }}+\mathcal{L}^{\mathrm{VF}+\text { Scalar }}
$$

where,

$$
\begin{aligned}
& \mathcal{L}^{\mathrm{VF}}= \bar{N}\left[i \gamma^{\mu}\left(\partial_{\mu}-i g \frac{\sigma^{a}}{2} W_{\mu}^{a}-i g^{\prime} \frac{Y^{\prime}}{2} B_{\mu}\right)-m_{N}\right] N \\
&+\overline{\chi_{1}}\left(i \gamma^{\mu} \partial_{\mu}-m_{\chi_{1}}\right) \chi_{1}-\left(Y_{1} \bar{N} \widetilde{H} \chi_{1}+\text { h.c. }\right) \\
&+\overline{\chi_{2}}\left(i \gamma^{\mu} \partial_{\mu}-m_{\chi_{2}}\right) \chi_{2}, \\
& \mathcal{L}^{\text {Scalar }}=\frac{1}{2} \partial^{\mu} S \partial_{\mu} S-\frac{1}{2} m_{S}^{2} S^{2}-\frac{1}{4 !} \lambda_{S} S^{4}-\frac{1}{2} \lambda_{S H}\left(H^{\dagger} H-\frac{v^{2}}{2}\right) S^{2},
\end{aligned}
$$

and

$$
\mathcal{L}^{\mathrm{VF}+\text { Scalar }}=-Y_{2}\left(\overline{\chi_{1}} \chi_{2} S+\text { h.c. }\right) \text {. }
$$

There are two Yukawa interactions present in this model. We will focus on the first in eq. (2.3). Electroweak symmetry breaking (EWSB) occurs as the SM Higgs acquires a vacuum expectation value: $H=\left(0 \frac{1}{\sqrt{2}}(v+h)\right)^{T}$ where $v=246 \mathrm{GeV}$. The Yukawa $Y_{1} \bar{N} \widetilde{H} \chi_{1}$ term in the Lagranigan (eq. (2.3)) mixes $N^{0}$ and $\chi_{1}$. Mass terms of the vector like fermions in $\mathcal{L}^{\mathrm{VF}}$ then take the following form:

$$
\begin{aligned}
-\mathcal{L}_{\text {mass }}^{\mathrm{VF}} & =m_{N} \overline{N^{0}} N^{0}+m_{N} N^{+} N^{-}+m_{\chi_{1}} \overline{\chi_{1}} \chi_{1}+\frac{Y_{1} v}{\sqrt{2}} \overline{N^{0}} \chi_{1}+\frac{Y_{1} v}{\sqrt{2}} \overline{\chi_{1}} N^{0} \\
& =\overline{\left(\chi_{1} N^{0}\right)}\left(\begin{array}{cc}
m_{\chi_{1}} & \frac{Y_{1} v}{\sqrt{2}} \\
\frac{Y_{1} v}{\sqrt{2}} & m_{N}
\end{array}\right)\left(\begin{array}{c}
\chi_{1} \\
N^{0}
\end{array}\right)+m_{N} N^{+} N^{-} \\
& \left.=\overline{\left(N_{1} N_{2}\right.}\right)\left(\begin{array}{cc}
m_{1} & 0 \\
0 & m_{2}
\end{array}\right)\left(\begin{array}{c}
N_{1} \\
N_{2}
\end{array}\right)+m_{N} N^{+} N^{-},
\end{aligned}
$$


where in the last step, the unphysical basis, $\left(\chi_{1} N^{0}\right)^{T}$ is related to physical basis, $\left(\begin{array}{ll}N_{1} & N_{2}\end{array}\right)^{T}$ through the following unitary transformation:

$$
\left(\begin{array}{c}
\chi_{1} \\
N^{0}
\end{array}\right)=\mathcal{U}\left(\begin{array}{l}
N_{1} \\
N_{2}
\end{array}\right)=\left(\begin{array}{cc}
\cos \theta & -\sin \theta \\
\sin \theta & \cos \theta
\end{array}\right)\left(\begin{array}{l}
N_{1} \\
N_{2}
\end{array}\right)
$$

where the mixing angle

$$
\tan 2 \theta=-\frac{\sqrt{2} Y_{1} v}{m_{N}-m_{\chi_{1}}} .
$$

The mass eigenvalues of the physical states $N_{1}$ and $N_{2}$, for small $\sin \theta(\sin \theta \rightarrow 0)$ limit, can be expressed as:

$$
\begin{aligned}
& m_{N_{1}} \simeq m_{\chi_{1}}+\frac{Y_{1} v}{\sqrt{2}} \sin 2 \theta \equiv m_{\chi_{1}}-\frac{\left(Y_{1} v\right)^{2}}{\left(m_{N}-m_{\chi_{1}}\right)} \\
& m_{N_{2}} \simeq m_{N}-\frac{Y_{1} v}{\sqrt{2}} \sin 2 \theta \equiv m_{N}+\frac{\left(Y_{1} v\right)^{2}}{\left(m_{N}-m_{\chi_{1}}\right)} .
\end{aligned}
$$

Here we have considered $Y_{1} v / \sqrt{2}<m_{\chi_{1}}<m_{N}$. Hence $m_{N_{1}}<m_{N_{2}}$. Therefore $N_{1}$ becomes the stable DM candidate (with a small kinematic caveat as we discuss shortly). Using eq. (2.8), one can find:

$$
\begin{aligned}
Y_{1} & =-\frac{\Delta m \sin 2 \theta}{\sqrt{2} v} \\
m_{N} & =m_{N_{1}} \sin ^{2} \theta+m_{N_{2}} \cos ^{2} \theta
\end{aligned}
$$

where $\Delta m=m_{N_{2}}-m_{N_{1}}$ is the mass difference between the two mass eigenstates and $m_{N}$ is the mass of electrically charged component of vectorlike fermion doublet $N^{\mp}$. This serves as an important parameter for the phenomenology of the model as we illustrate. Note again that due to a different $\mathcal{Z}_{2} \times \mathcal{Z}_{2}^{\prime}$ charge, $\chi_{2}$ do not mix with $N$ and $\chi_{1}$.

Vector like fermion DM has gauge interactions to SM due to the inclusion of doublet in the model. Expanding the covariant derivative in $\mathcal{L}^{\mathrm{VF}}$, one can find:

$$
\begin{aligned}
\mathcal{L}_{\text {int }}^{\mathrm{VF}}= & \bar{N} i \gamma^{\mu}\left(-i g \frac{\sigma^{a}}{2} W_{\mu}^{a}+i \frac{g^{\prime}}{2} B_{\mu}\right) N \\
= & \left(\frac{e_{0}}{2 \sin \theta_{W} \cos \theta_{W}}\right) \overline{N^{0}} \gamma^{\mu} Z_{\mu} N^{0}+\frac{e_{0}}{\sqrt{2} \sin \theta_{W}} \overline{N^{0}} \gamma^{\mu} W_{\mu}^{+} N^{-}+\frac{e_{0}}{\sqrt{2} \sin \theta_{W}} N^{+} \gamma^{\mu} W_{\mu}^{-} N^{0} \\
& -e_{0} N^{+} \gamma^{\mu} A_{\mu} N^{-}-\left(\frac{e_{0}}{2 \sin \theta_{W} \cos \theta_{W}}\right) \cos 2 \theta_{W} N^{+} \gamma^{\mu} Z_{\mu} N^{-}
\end{aligned}
$$

where $g=e_{0} / \sin \theta_{W}$ and $g^{\prime}=e_{0} / \cos \theta_{W}$ with $e_{0}$ being the electromagnetic coupling constant and $\theta_{W}$ being the Weinberg angle. One can therefore express the gauge and the 
Yukawa interactions of $\mathcal{L}^{\mathrm{VF}}$ in mass basis of $N_{1}$ and $N_{2}$ as:

$$
\begin{aligned}
& \mathcal{L}_{\text {int }}^{\mathrm{VF}}=\left(\frac{e_{0}}{2 \sin \theta_{W} \cos \theta_{W}}\right)\left[\sin ^{2} \theta \overline{N_{1}} \gamma^{\mu} Z_{\mu} N_{1}+\cos ^{2} \theta \overline{N_{2}} \gamma^{\mu} Z_{\mu} N_{2}\right. \\
& \left.+\sin \theta \cos \theta\left(\overline{N_{1}} \gamma^{\mu} Z_{\mu} N_{2}+\overline{N_{2}} \gamma^{\mu} Z_{\mu} N_{1}\right)\right] \\
& +\frac{e_{0}}{\sqrt{2} \sin \theta_{W}} \sin \theta \overline{N_{1}} \gamma^{\mu} W_{\mu}^{+} N^{-}+\frac{e_{0}}{\sqrt{2} \sin \theta_{W}} \cos \theta \overline{N_{2}} \gamma^{\mu} W_{\mu}^{+} N^{-} \\
& +\frac{e_{0}}{\sqrt{2} \sin \theta_{W}} \sin \theta N^{+} \gamma^{\mu} W_{\mu}^{-} N_{1}+\frac{e_{0}}{\sqrt{2} \sin \theta_{W}} \cos \theta N^{+} \gamma^{\mu} W_{\mu}^{-} N_{2} \\
& -e_{0} N^{+} \gamma^{\mu} A_{\mu} N^{-}-\left(\frac{e_{0}}{2 \sin \theta_{W} \cos \theta_{W}}\right) \cos 2 \theta_{W} N^{+} \gamma^{\mu} Z_{\mu} N^{-} \\
& -\frac{Y_{1}}{\sqrt{2}} h\left[\sin 2 \theta\left(\overline{N_{1}} N_{1}-\overline{N_{2}} N_{2}\right)+\cos 2 \theta\left(\overline{N_{1}} N_{2}+\overline{N_{2}} N_{1}\right)\right]
\end{aligned}
$$

Let us now focus into the other Yukawa interaction between different DM particles as introduced in $\mathcal{L}^{\mathrm{VF}+\mathrm{Scalar}}$ (eq. (2.5)). In the physical basis it reads:

$$
\mathcal{L}_{\text {int }}^{\mathrm{VF}+\text { Scalar }}=-Y_{2}\left(\cos \theta \overline{N_{1}} \chi_{2} S-\sin \theta \overline{N_{2}} \chi_{2} S+\cos \theta \overline{\chi_{2}} N_{1} S-\sin \theta \overline{\chi_{2}} N_{2} S\right) .
$$

The scalar field $S$ do not acquire any vev and thus retains the $\mathcal{Z}_{2} \times \mathcal{Z}^{\prime}{ }_{2}$ symmetry intact and is eligible as a possible DM candidate of the model. The interaction terms involving $S$ of $\mathcal{L}^{\text {Scalar }}$ after EWSB turns out to be:

$$
\mathcal{L}_{\text {int }}^{\text {Scalar }}=-\frac{\lambda_{S H} v}{2} h S^{2}-\frac{\lambda_{S H}}{4} h^{2} S^{2} .
$$

Following $\mathcal{L}^{\text {Scalar }}$ (eq. (2.4)), the full scalar potential including SM Higgs can be written as:

$$
V(H, S)=-\mu_{H}^{2}\left(H^{\dagger} H\right)+\lambda_{H}\left(H^{\dagger} H\right)^{2}+\frac{1}{2} m_{S}^{2} S^{2}+\frac{\lambda_{S}}{4 !} S^{4}+\frac{\lambda_{S H}}{2}\left(H^{\dagger} H-\frac{v^{2}}{2}\right) S^{2} .
$$

It is important now to identify the key parameters of the model which control relevant phenomenology of the model. Mainly seven independent parameters do the job including two DM masses, mass of the mediator, mixing angle of singlet-doublet fermion, Yukawa coupling denoting DM-DM interactions and the Higgs portal coupling of the scalar DM respectively:

$$
\left\{m_{N_{1}}, \Delta m, m_{S}, m_{\chi_{2}}, \sin \theta, Y_{2}, \lambda_{S H}\right\}
$$

\subsection{Constraints on the model parameters}

Before evaluating the constraints on the model parameters given in eq. (2.16), from DM and collider analysis, we would like to go through the constraints coming from stability of the potential, perturbativity of the parameters and invisible decay widths of $Z$ and $H$ to determine the broad parameter space available for our numerical scan.

- Stability of potential: for the tree-level vacuum stability of the scalar potential as in eq. (2.15), one requires to satisfy the following co-positivity conditions [41]:

$$
\lambda_{H} \geq 0, \quad \lambda_{S} \geq 0, \quad \text { and } \quad \lambda_{\mathrm{SH}}+\sqrt{\frac{2}{3} \lambda_{\mathrm{H}} \lambda_{\mathrm{S}}} \geq 0 .
$$


This essentially means, we satisfy the constraints if we assume $\lambda_{H}, \lambda_{S}, \lambda_{S H} \geq 0$ throughout the scan.

- Perturbativity: the upper limit of perturbativity bound on quartic and Yukawa couplings of the model are given by,

$$
\begin{array}{r}
\left|\lambda_{S}\right|,\left|\lambda_{S H}\right|<4 \pi \\
\text { and }\left|\mathrm{Y}_{1}\right|,\left|\mathrm{Y}_{2}\right|<\sqrt{4 \pi}
\end{array}
$$

- Relic density of DM: the total relic density of DM is limited by the combined WMAP [5] and PLANCK [6] data as:

$$
\Omega_{\mathrm{DM}} h^{2}=0.1161 \pm 0.0028 .
$$

- Invisible decay width of Higgs: invisible Higgs decay width puts strong constraints on light DM having masses $<m_{h} / 2$ if they are connected through Higgs portal, as we have in the model. Current bound from LHC on Higgs invisible branching fraction is given by [42]:

$$
\operatorname{Br}(\text { Higgs } \rightarrow \text { inv. })<0.24 \text {. }
$$

Details have been furnished in appendix B.

- Invisible decay width of Z: Z boson can decay to DM with $m_{D M}<M_{Z} / 2$, whenever the DM has a weak charge as is the case for the fermion DM component of the model. Invisible decay of $Z$ is strongly constrained from observable data. The upper limit of invisible $\mathrm{Z}$ decay width is [42]:

$$
\Gamma(Z \rightarrow \text { inv. }) \leq 499 \pm 1.5 \mathrm{MeV} .
$$

One may find the details about this constraint applied to our case in appendix C.

\subsection{Possible multipartite DM scenarios}

We have four electromagnetic charge neutral particles in the model: $N^{0}, \chi_{1}, \chi_{2}$ and $S$. Given the same charge of $N^{0}$ and $\chi_{1}$ under $\mathcal{Z}_{2} \times \mathcal{Z}_{2}{ }^{\prime}$, they mix and the lighter eigenstate $N_{1}$ (with $m_{N_{1}}<m_{N_{2}}$ ) can not decay to SM, while $N_{2}$ decays to $N_{1}$. Then, we are left with three possible DM candidates, i.e. $N_{1}, \chi_{2}$ and $S$. However, the absolute stability will be dictated by other Yukawa coupling present in dark sector $Y_{2} \overline{\chi_{1}} \chi_{2} S \rightarrow Y_{2} \overline{N_{1}} \chi_{2} S$ (as in eq. (2.13)). Evidently, if one of the physical states is heavier than the other two, then it can decay to the other two lighter particles and become unstable. As a result, the two lighter physical states will be the viable DM candidates. Therefore, depending on the mass hierarchy, the model offers four different types of multipartite DM scenarios as illustrated in figure 2.

- Type-I: $m_{\chi_{2}}>m_{N_{1}}+m_{S}: \quad N_{1}$ and $S$ are the stable DM components.

- Type-II: $m_{S}>m_{N_{1}}+m_{\chi_{2}}: N_{1}$ and $\chi_{2}$ are stable DM components.

- Type-III: $m_{N_{1}}>m_{S}+m_{\chi_{2}}: S$ and $\chi_{2}$ are stable DM components. 


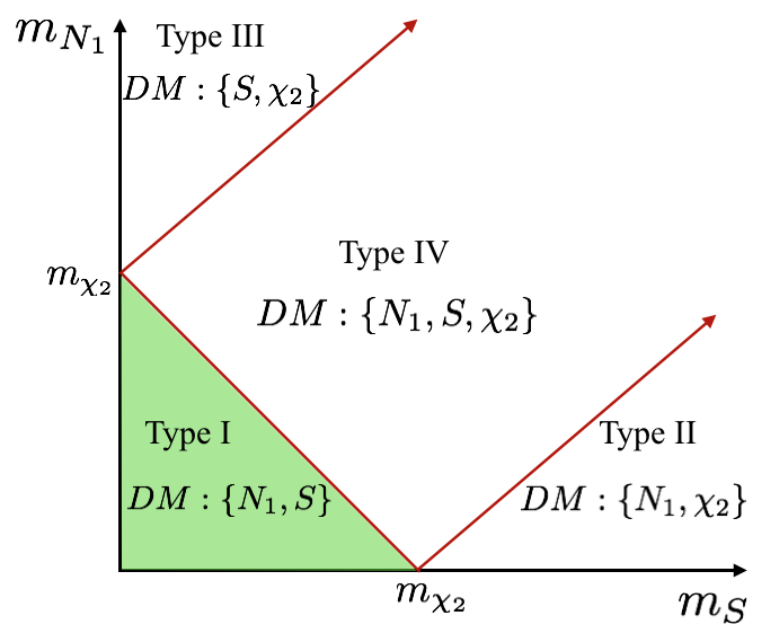

Figure 2. Different types of multicomponent DM scenarios that can be realised in the model depicted in $m_{N_{1}}-m_{S}$ plane, given that a hierarchy among $m_{N_{1}}, m_{S}, m_{\chi_{2}}$. Type-I scenario (coloured in green) is analysed in this paper.

- Type-IV: if $m_{\chi_{2}}<m_{N_{1}}+m_{S}, m_{S}<m_{N_{1}}+m_{\chi_{2}}$ and $m_{N_{1}}<m_{S}+m_{\chi_{2}}$, then all three particles $N_{1}, \chi_{2}$ and $S$ are stable and will yield a three-component DM scenario.

In this paper, we focus mostly on Type-I scenario (green region in figure 2). This gives us an opportunity to compare with the single component cases of the corresponding DM components $\left(N_{1}\right.$ and $S$ ), which are very well studied, and indicate the effects of DM-DM conversion employed in this set-up.

\section{Review of single component DM frameworks with $N_{1}$ and $S$}

Before we discuss the two component DM set up (of Type-I) as advocated above, we need to know the fate of the individual DMs in single component frameworks. We review relic density and direct search allowed parameter space for both vectorlike fermion $\operatorname{DM}\left(N_{1}\right)$ and singlet scalar DM $(S)$ in the next two consecutive subsections.

\subsection{Single component fermion DM $\left(N_{1}\right)$}

The presence of vector-like fermion singlet $\left(\chi_{1}\right)$ and a doublet $(N)$ can give rise to a fermion DM [34], where both transform under a $\mathcal{Z}_{2}$ symmetry. The relevant Lagrangian is still given by $\mathcal{L}^{\mathrm{VF}}$ as in eq. (2.3). As described above, the singlet and the neutral component of the doublet mix after EWSB, and the lightest component of the neutral physical states $N_{1}$ becomes a stable single component DM.

We note here that the freeze-out abundance of $N_{1} \mathrm{DM}$ is controlled by the annihilation and co-annihilation channels as detailed in appendix A (figures 23, 24 and 25). Therefore, the important parameters which decide the relic abundance of $N_{1}$ are

$$
\left\{m_{N_{1}}, \Delta m, \sin \theta\right\} .
$$




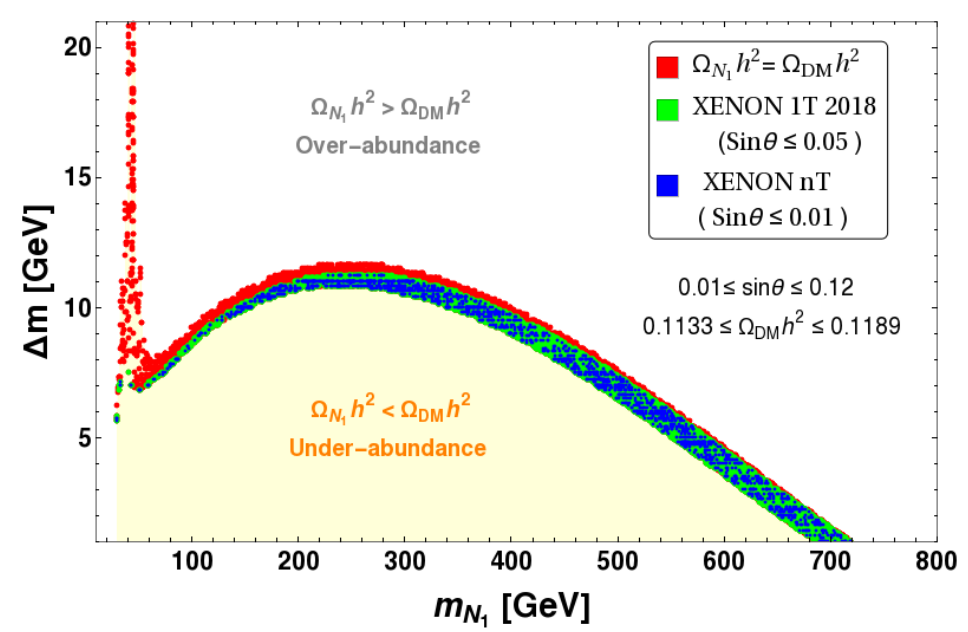

Figure 3. Relic density allowed (red points) and direct detection \{XENON 1T (green points), XENON $\mathrm{nT}$ (blue points) $\}$ allowed parameter space for single component fermion $\mathrm{DM}\left(N_{1}\right)$ is shown in $m_{N_{1}}-\Delta m$ plane. Under abundance $\left(\Omega_{N_{1}} h^{2}<\Omega_{D M} h^{2}\right)$ (yellow region below the red points) and over-abundance $\left(\Omega_{N_{1}} h^{2}>\Omega_{D M} h^{2}\right)$ (the region above the red points) are also indicated. $\Omega_{D M} h^{2}$ range is mentioned in the figure inset and also in eq. (2.19).

Due to singlet-doublet mixing, the DM in direct search experiments can scatter off the target nucleus via both $Z$ and Higgs mediated processes (shown in top pannel of figure 9).

The relic density and direct search allowed parameter space for $N_{1} \mathrm{DM}$ is shown in figure 3. This is shown in $m_{N_{1}}-\Delta m$ plane for small values of $\sin \theta$. It has already been noted [34] that due to $Z$ mediation, $\sin \theta$ is limited to very small values $\lesssim 0.1$ by the non-observation of DM in direct search experiments. We therefore choose only such small mixing regions for illustration in figure 3. Essentially, the whole relic density allowed plane is also allowed by direct search constraints (XENON1T [10], XENON nT [11] as shown in the figure 3). The under and over abundant regions are also indicated, which will be more useful for discussing the two component framework. The important message from this graph is that for small $\sin \theta, \Delta m$ has to be small $(\lesssim 12 \mathrm{GeV})$ to satisfy relic density, except for those low DM mass resonance regions $\left(\sim m_{Z} / 2, m_{h} / 2\right)$. For under abundance, the DM has to obtain even higher annihilation cross-section. For small $\sin \theta$ to satisfy direct search, the only way to probe under abundant regions is to have even smaller $\Delta m$ to enhance coannihilation effects. Therefore, when we embed the fermion DM in a non-interacting two component DM framework, the under abundant regions (as indicated in figure 3) are going to be allowed. However, the situation alters in presence of an interacting two-component framework as we will demonstrate in section 4 .

\subsection{Single component scalar DM $(S)$}

The Lagrangian $\mathcal{L}^{\text {Scalar }}$ in eq. (2.4), describes the case of single component scalar DM $S$. The relevant parameters describing the scalar DM interaction with SM is given by

$$
\left\{m_{S}, \lambda_{S H}\right\} .
$$

The annihilation process which controls the freeze-out of $S$ are shown in figure 4. 

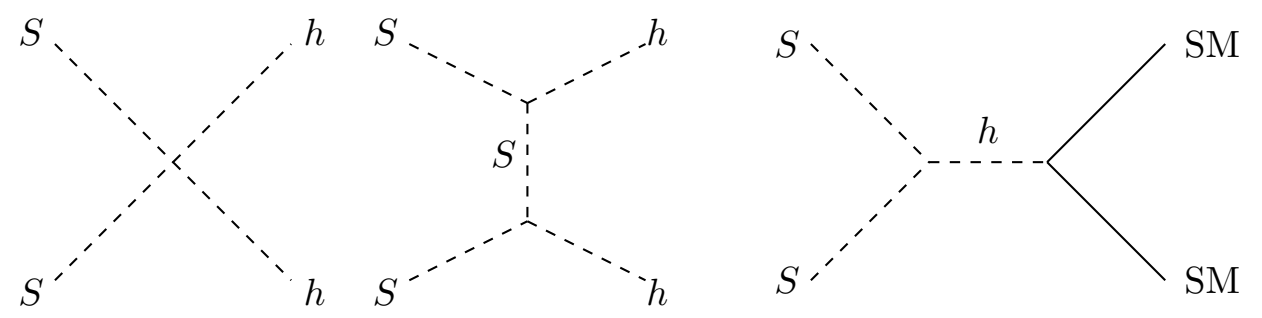

Figure 4. Feynman diagrams for Scalar DM $S$ annihilating to SM particles i.e $S S \rightarrow$ SM SM.

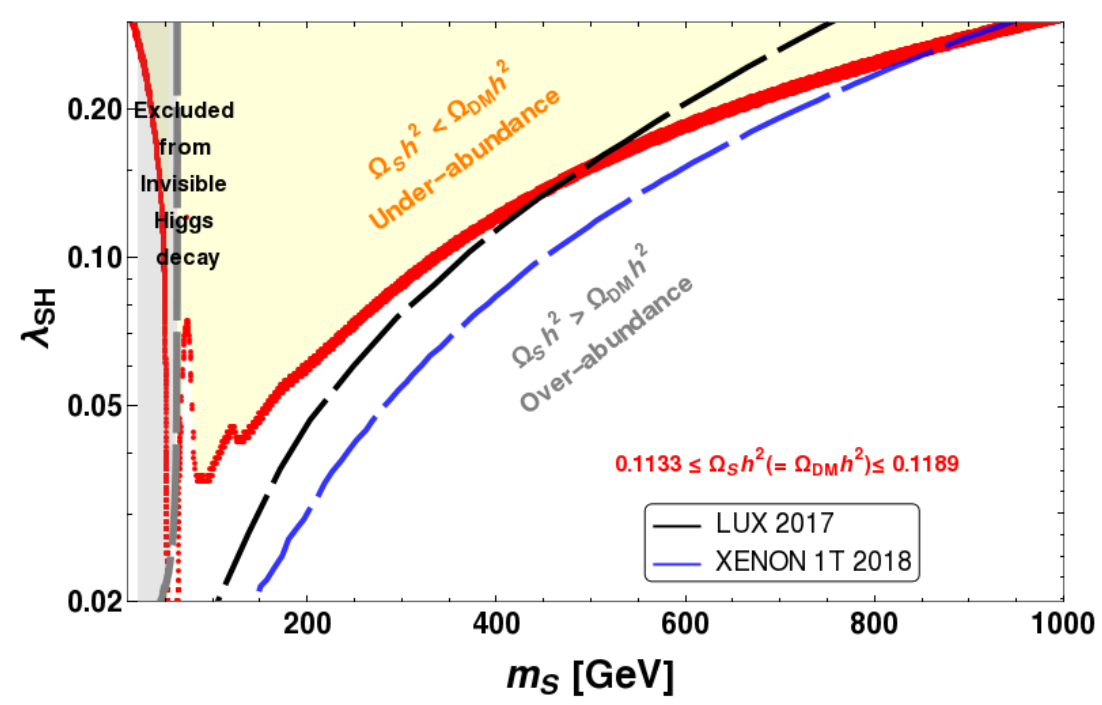

Figure 5. Relic density allowed $\left(\Omega_{S} h^{2}=\Omega_{D M} h^{2}\right)$ (red region) parameter space for scalar DM $(S)$ is shown in $m_{S}-\lambda_{S H}$ plane. LUX [9] (black dashed) and XENON 1T [10] (blue dashed) exclusion limits are also shown. The region 'above' the red patch (in yellow) corresponds to under abundance $\left(\Omega_{S} h^{2}<\Omega_{D M} h^{2}\right)$ and the one below corresponds to over abundance $\left(\Omega_{S} h^{2}>\Omega_{D M} h^{2}\right)$. Exclusion limit from invisible Higgs decays is shown by grey region. $\Omega_{D M} h^{2}$ range is mentioned in the figure inset and also in eq. (2.19).

The relic density allowed parameter space for the scalar DM is well studied [20, 21, 32, 33,37 and is summarised in figure 5 , in terms of DM mass $\left(m_{S}\right)$ and Higgs portal coupling $\left(\lambda_{S H}\right)$. Direct search sensitivities of LUX [9] and XENON 1T [10] from null detection are also shown in the same graph for $S$ which only has a $t$-channel Higgs mediation with nucleus (shown in bottom pannel of figure 9). This essentially shows that if $S$ contributes to the full DM relic density, it lives either in resonance region $\left(m_{S} \sim m_{h} / 2\right)$ or in high DM mass regions $\left(m_{S} \gtrsim 900 \mathrm{GeV}\right)$ to satisfy null observations from direct search experiments. Under abundance for $S$ can only be achieved with larger annihilation cross-section, that can only occur with larger Higgs portal coupling $\left(\lambda_{S H}\right)$ and that is even more constrained from direct search data. If the scalar DM is embedded in an non-interacting multi-component DM framework, it is further restricted by direct search, discarding $m_{S}$ upto $\mathrm{TeV}$ or more. We will show in section 4 , that the situation alters in presence of an additional DM component, with which the scalar DM has non-negligible interactions. We also point out that the 

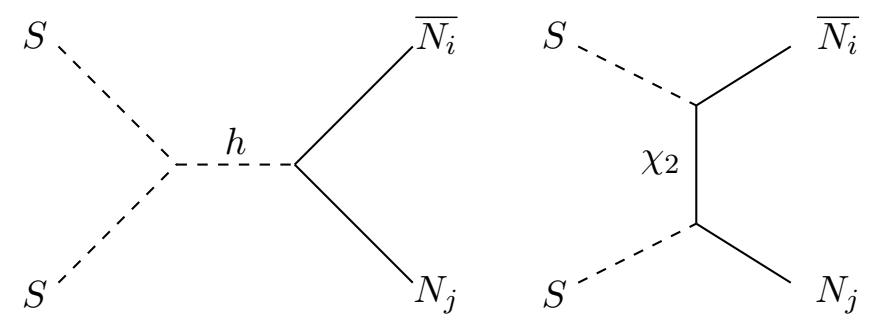

Figure 6. Diagrams contributing to DM-DM conversion $(i=1,2)$ between fermion $\left(N_{i}\right)$ and scalar $\mathrm{DM}(S)$ components.

presence of a heavy scalar $S_{H}$ (also a SM singlet) having same $\mathcal{Z}_{2}$ charge as of $S$, can change the conclusion significantly allowing a larger parameter space through co-annihilation (in section 5).

\section{Two component DM with $N_{1}$ and $S$}

As already discussed in section 2.2, we choose Type-I case for illustrating a two-component interacting DM model with $m_{\chi_{2}}>m_{N_{1}}+m_{S}$, where $N_{1}$ froms a vectorlike fermion DM component and $S$ forms a scalar DM component. The heaviest field $\chi_{2}$ in the dark sector, which can decay to $N_{1}$ and $S$, act as a mediator between the two DM components through the Yukawa interaction: $Y_{2} \overline{\chi_{2}} \chi_{1} S$. These DMs can also interact with each other through Higgs portal couplings: $Y_{1} \bar{N} \widetilde{H} \chi_{1}$ and $\lambda_{S H}\left(H^{\dagger} H\right) S^{2}$. The DM-DM interactions of this model is shown by the Feynman diagrams in figure 6 .

DM-DM conversion diagrams will dominantly help the heavier DM component to annihilate into the lighter one and therefore contribute to its thermal freeze-out and relic density. Apart from DM masses and mediator mass $\left(m_{\chi_{2}}\right)$, the DM-DM conversion is a function of the following couplings

$$
\left\{Y_{1}, Y_{2}, \lambda_{S H}\right\}
$$

However, the Higgs portal couplings $Y_{1}$ (as a function of $\Delta m$ and $\sin \theta$, see in eq. (2.10)) and $\lambda_{S H}$ are strongly constrained from direct detection bound (already discussed in section 3 ). Therefore, DM-DM interaction through Higgs mediation will be negligible in relic density and direct search allowed parameter space of the two component model and can be identified with $Y_{2}=0$ situation. We will show that in such a case, the two DMs are almost decoupled and behave like single component cases to occupy the under abundant regions of their corresponding DM parameter space. Here lies the importance of assuming the presence of a heavy mediator $\chi_{2}$ in this model to carry out DM-DM interactions through Yukawa coupling $Y_{2}$.

\subsection{Coupled Boltzmann equations}

The thermal freeze-out of two component DM framework is described by a coupled Boltzmann equations (BEQs) and can be written as a function of reduced $x$, where $x=\mu / T$, 
with $\frac{1}{\mu}=\frac{1}{m_{N_{1}}}+\frac{1}{m_{N_{2}}}+\frac{1}{m_{S}}[20,25,43]$. The one here reads:

$$
\begin{aligned}
& \frac{d Y_{N_{i}}}{d x}=-0.264 M_{\mathrm{Pl}} \sqrt{g}_{*} \frac{\mu}{x^{2}}\left[\sum _ { j } \left\{\left\langle\sigma v_{\overline{N_{i}} N_{j} \rightarrow S M}\right\rangle\left(Y_{N_{i}} Y_{N_{j}}-Y_{N_{i}}^{\mathrm{EQ}} Y_{N_{j}}^{\mathrm{EQ}}\right)\right.\right. \\
& +\left\langle\sigma v_{\overline{N_{i}} N_{j} \rightarrow S S}\right\rangle\left(Y_{N_{i}} Y_{N_{j}}-\frac{Y_{N_{i}}^{\mathrm{EQ}} Y_{N_{j}}^{\mathrm{EQ}}}{Y_{S}^{\mathrm{EQ}}} Y_{S}^{2}\right) \Theta\left(m_{N_{i}}+m_{N_{j}}-2 m_{S}\right) \\
& \left.-\left\langle\sigma v_{S S \rightarrow \overline{N_{i}} N_{j}}\right\rangle\left(Y_{S}^{2}-\frac{Y_{S}^{\mathrm{EQ}}}{Y_{N_{i}}^{\mathrm{EQ}} Y_{N_{j}}^{\mathrm{EQ}}} Y_{N_{i}} Y_{N_{j}}\right) \Theta\left(2 m_{S}-m_{N_{i}}-m_{N_{j}}\right)\right\} \\
& \left.+\left\langle\sigma v_{\overline{N_{i}} N^{ \pm} \rightarrow S M}\right\rangle\left(Y_{N_{i}} Y_{N^{ \pm}}-Y_{N_{i}}^{\mathrm{EQ}} Y_{N^{ \pm}}^{\mathrm{EQ}}\right)\right], \\
& \frac{d Y_{S}}{d x}=-0.264 M_{\mathrm{Pl}} \sqrt{g}_{*} \frac{\mu}{x^{2}}\left[\left\langle\sigma v_{S S \rightarrow S M}\right\rangle\left(Y_{S}^{2}-Y_{S}^{\mathrm{EQ}}{ }^{2}\right)\right. \\
& +\sum_{i, j}\left\{-\left\langle\sigma v_{\overline{N_{i}} N_{j} \rightarrow S S}\right\rangle\left(Y_{N_{i}} Y_{N_{j}}-\frac{Y_{N_{i}}^{\mathrm{EQ}} Y_{N_{j}}^{\mathrm{EQ}}}{Y_{S}^{\mathrm{EQ}}} Y_{S}^{2}\right) \Theta\left(m_{N_{i}}+m_{N_{j}}-2 m_{S}\right)\right. \\
& \left.\left.+\left\langle\sigma v_{S S \rightarrow \overline{N_{i}} N_{j}}\right\rangle\left(Y_{S}^{2}-\frac{Y_{S}^{\mathrm{EQ}}}{Y_{N_{i}}^{\mathrm{EQ}} Y_{N_{j}}^{\mathrm{EQ}}} Y_{N_{i}} Y_{N_{j}}\right) \Theta\left(2 m_{S}-m_{N_{i}}-m_{N_{j}}\right)\right\}\right],
\end{aligned}
$$

where the subscripts $i, j=1,2$ describes the fermion DM and the heavy neutral fermion component of the model respectively. In the above equations, we note that the annihilation contribution of $N_{i}$ to $S$ or otherwise depending on the mass hierarchy is included. The equilibrium distributions now recast in terms of $\mu$ takes the form:

$$
\begin{aligned}
& Y_{N_{i}}^{\mathrm{EQ}}(x)=0.145 \frac{g}{g_{*}} x^{\frac{3}{2}}\left(\frac{m_{i}}{\mu}\right)^{\frac{3}{2}} e^{-x\left(\frac{m_{i}}{\mu}\right)} \\
& Y_{S}^{\mathrm{EQ}}(x)=0.145 \frac{g}{g_{*}} x^{\frac{3}{2}}\left(\frac{m_{S}}{\mu}\right)^{\frac{3}{2}} e^{-x\left(\frac{m_{S}}{\mu}\right)}
\end{aligned}
$$

The relic density allowed parameter space of the two-component framework is then given by the solution of the above Boltzmann equations, that determine the freeze-out of the individual components depending on annihilations plus co-annihilations and DM-DM interactions. Obviously, total DM relic density for the two component case will be the sum of individual relic density as:

$$
\Omega_{T} h^{2}=\Omega_{N_{1}} h^{2}+\Omega_{S} h^{2},
$$

which should satisfy combined WMAP and PLANCK limit $0.1133 \leq \Omega_{T} h^{2}\left(=\Omega_{D M} h^{2}\right) \leq$ 0.1189 [6]. Individual relic density in interacting multipartite DM case can be found out by numerical solution to the coupled Boltzmann equations or approximate analytical solution of coupled BEQ [20] and that of the $i$-th DM candidate is given by:

$$
\Omega_{i} h^{2}=\frac{854.45 \times 10^{-13}}{\sqrt{g_{*}}} \frac{x_{f}^{i}}{\langle\sigma v\rangle_{i}^{T}},
$$


where $\langle\sigma v\rangle_{i}^{T}$ is the total effective annihilation cross-section and $x_{f}^{i}$ corresponds to freeze-out temperature of the $i$ th DM component. Note however, for the ease of the analysis, we are not using the approximate solution here; relic density and direct search cross-sections for both the DM components are obtained numerically by inserting the model in MicrOmegas package [44].

If fermion DM is heavier than scalar DM $\left(m_{N_{1}}>m_{S}\right)$, then heavier DM component $\left(N_{1}\right)$ can annihilate to lighter component $(S)$ following processes as in figure 6 . Such DM-DM conversion affects the freeze out of heavier DM component and hence its relic density [20]. The lighter DM candidate on the other hand, have no new channel to deplete its number density and behave almost like single component DM. Then $\langle\sigma v\rangle_{N_{1}}^{T}$ for fermionic DM assuming $m_{N_{1}}>m_{S}$ will be given by:

$$
\langle\sigma v\rangle_{N_{1}}^{T} \simeq\langle\sigma v\rangle_{N_{1}}^{\mathrm{eff}}+\langle\sigma v\rangle_{\bar{N}_{1} N_{1} \rightarrow S S}+2\langle\sigma v\rangle_{\bar{N}_{1} N_{2} \rightarrow S S}\left(1+\frac{\Delta m}{m_{N_{1}}}\right)^{3 / 2} e^{\frac{-\Delta m}{T}}
$$

where $\langle\sigma v\rangle_{N_{1}}^{\text {eff }}$ is the annihilation plus co-annihilation cross-section of fermion DM to SM given by eq. (A.1). The last term in the above equation represents co-annihilation to scalar DM component and is therefore aided by the Boltzmann factor along with a symmetry factor of 2 (assuming $m_{N_{1}} \simeq m_{N_{2}}$ ). In this limit of $m_{N_{1}}>m_{S}$, the annihilation cross-section for scalar DM $(S)$ only captures the annihilations to SM as in a single component framework:

$$
\langle\sigma v\rangle_{S}^{T}=\langle\sigma v\rangle_{S S \rightarrow S M S M}
$$

Evidently, for the opposite hierarchy, $m_{S}>m_{N_{1}}$ :

$$
\begin{aligned}
\langle\sigma v\rangle_{N_{1}}^{T} & =\langle\sigma v\rangle_{N_{1}}^{\mathrm{eff}}, \\
\langle\sigma v\rangle_{S}^{T} & =\langle\sigma v\rangle_{S S \rightarrow S M S M}+\langle\sigma v\rangle_{S S \rightarrow \overline{N_{i}} N_{j}} .
\end{aligned}
$$

We note here that the relic density of the lighter DM may also get affected by DM-DM conversion when the production from the heavier component becomes significantly large and comparable to its annihilation to SM. This is to remind again that the parameter space scan performed in the subsequent analysis do not use approximate solutions to the coupled BEQs derived above but uses the numerical results obtained from the code MicrOmegas which nicely captures all the features of individual DM relic density affected by DM-DM conversion.

\subsection{Relic density and direct search outcome}

We first study the variation of individual relic densities with corresponding DM masses as shown in figure 7. Two possible mass hierarchies are shown; in top we choose $m_{S}>m_{N_{1}}$ and in the bottom panel we have $m_{S}<m_{N_{1}}$. Relic density of fermion DM $\left(N_{1}\right)$ is shown in the left panel and that of the scalar $(S)$ is shown in the right panel. We see that for $m_{S}>m_{N_{1}}$ (top left panel of figure 7 ), $\Omega_{N_{1}} h^{2}$ do not change with different choices of Yukawa coupling $Y_{2}$. However with same hierarchy $\left(m_{S}>m_{N_{1}}\right)$ for $S$, relic density is steadily reduced with larger choice of $Y_{2}$ (top right panel). It is exactly the other way 

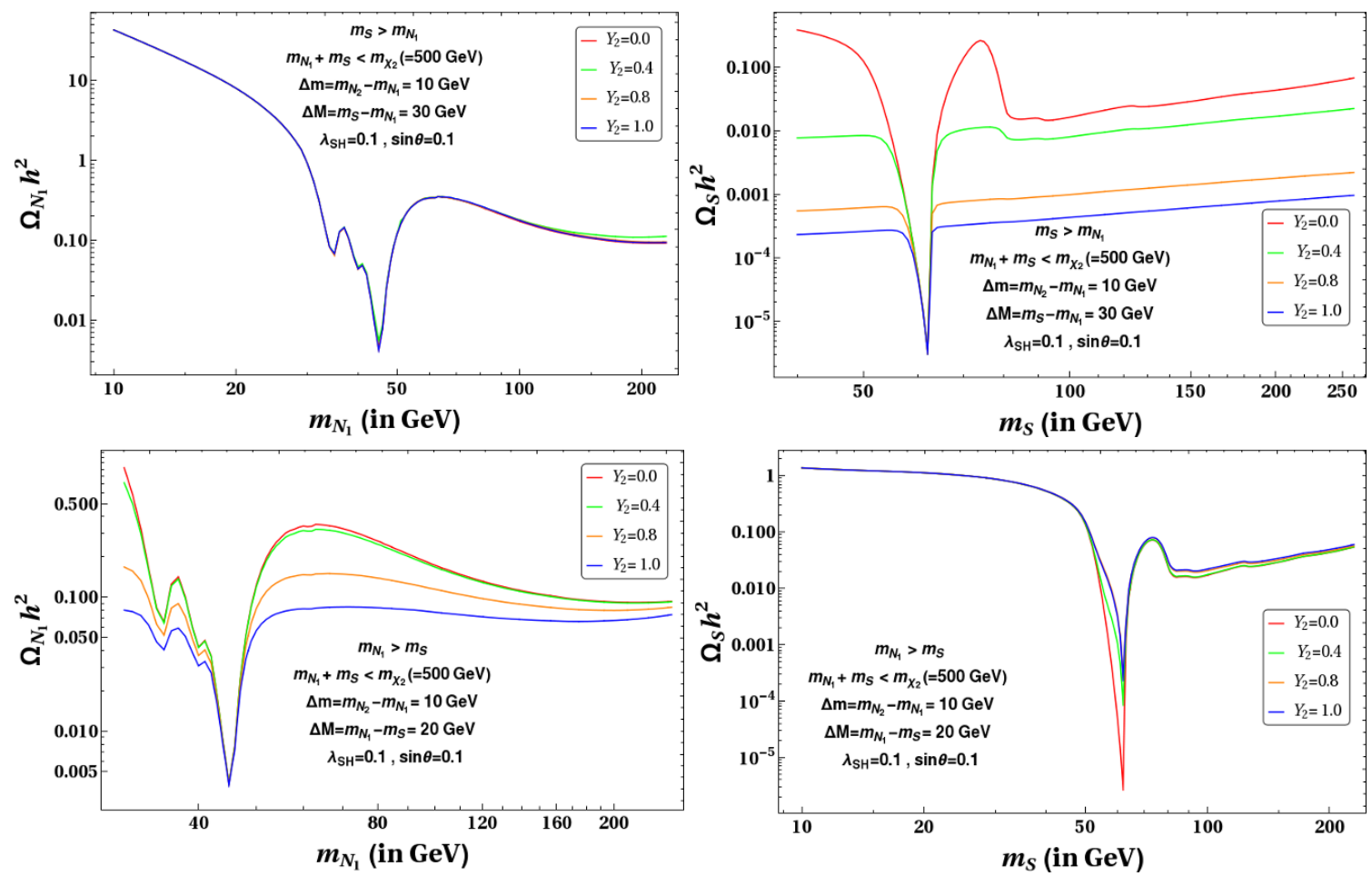

Figure 7. Relic densities $\left(\Omega_{N_{1}} h^{2}\right.$ and $\left.\Omega_{\mathrm{S}} \mathrm{h}^{2}\right)$ of the individual DM components as a function of respective DM masses $\left(m_{N_{1}}\right.$ and $\left.\mathrm{m}_{\mathrm{s}}\right)$ shown in left and right panel respectively. Two possible mass hierarchies are shown: $m_{S}>m_{N_{1}}$ (top panel) and $m_{N_{1}}>m_{S}$ (bottom panel). Different values of $Y_{2}=0.0$ (red), 0.4 (green), 0.8 (orange), 1.0 (blue) are chosen keeping other parameters fixed (as mentioned in the plots) to decipher DM-DM interactions.

round, when we have $m_{S}<m_{N_{1}}$ (bottom panel of figure 7). In such a case, relic density for $N_{1}$ decreases with larger $Y_{2}$, while it remains unaltered for $S$. This follows from the analytic solution of the effective annihilation cross sections as mentioned in eqs. (4.5), (4.6), (4.7) showing the importance of DM-DM conversion. In this plot we have kept other parameters fixed as mentioned in the plot, particularly with a moderate value of the mediator mass fixed at $m_{\chi_{2}}=500 \mathrm{GeV}$.

The sensitivity of individual relic densities to DM-DM conversion as a function of mediator mass $\left(m_{\chi_{2}}\right)$ is shown in figure 8. Evidently, we demonstrate it for the heavier component ( $N_{1}$ on the left and $S$ on the right) with different choices of mediator masses: $m_{\chi_{2}}: 500$ (red), 1000 (green) and 2000 (blue) GeV, keeping $Y_{2}=1.0$ and $\lambda_{S H}=0.1$ fixed. In the same figure 8 , we have also demonstrated the case of $Y_{2}=0$ (purple dotted line), when $\chi_{2}$ does not take part in the DM-DM conversions. It is evident that with large $m_{\chi_{2}}$, DM-DM conversion becomes feeble and closely resembles $Y_{2}=0$ (purple dotted line) case. Therefore, large Yukawa $Y_{2}$ can play an important role in relic density, but with not-so-heavy mediator mass $\left(m_{\chi_{2}}\right)$. The lighter DM component is again mostly unaffected by DM-DM conversion as has already been discussed. One important point to note is the difference between $Y_{2}=1.0$ and $\lambda_{S H}=0.1$ chosen for illustration. This is because $Y_{2}$ remains unconstrained (excepting for large perturbative limit $\leq \sqrt{4 \pi}$ ), while $\lambda_{S H}$ is 

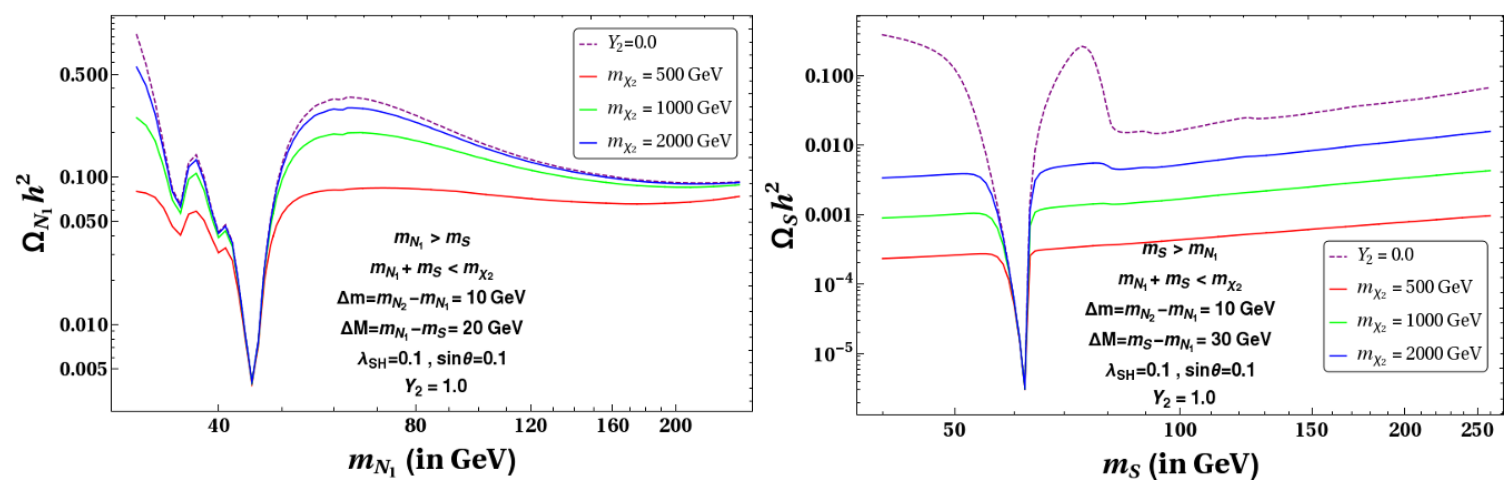

Figure 8. Sensitivity of mediator mass $\left(m_{\chi_{2}}\right)$ to DM-DM conversion and that to relic density of the heavier component is demonstrated. [Left] $\Omega_{N_{1}} h^{2}$ as a function of $m_{N_{1}}$ for different values of $m_{\chi_{2}}=500$ (red), 1000 (green), $2000 \mathrm{GeV}$ (blue) assuming $m_{N_{1}}>m_{S}$. [Right] $\Omega_{S} h^{2}$ as a function of $m_{S}$ for $m_{S}>m_{N_{1}}$. Other parameters kept fixed at different values are mentioned in the plot along with $Y_{2}=1.0$ and $\lambda_{S H}=0.1$. Purple dotted line in both graphs correspond to $Y_{2}=0$ case, shown for comparison.

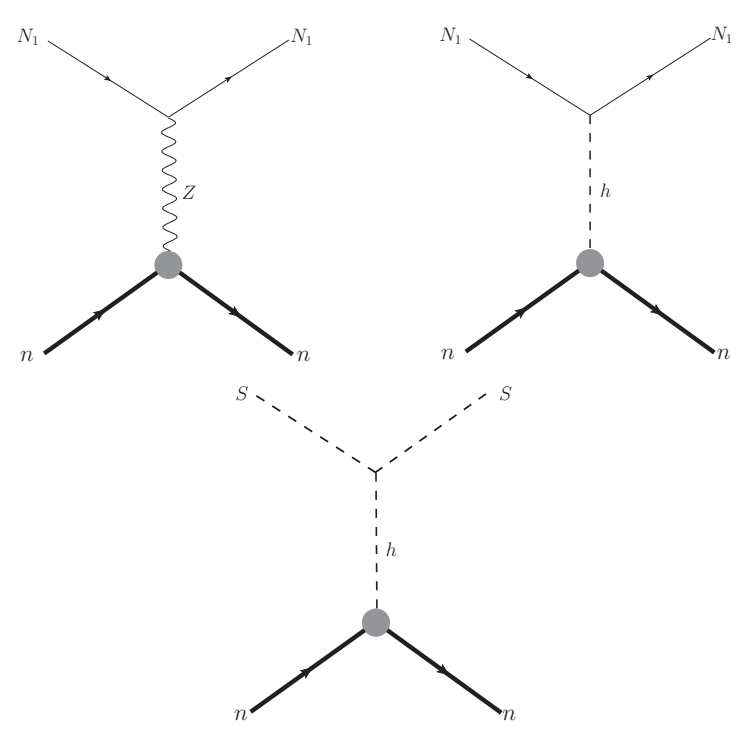

Figure 9. Feynman diagrams of spin independent (SI) direct detection of fermion DM (top panel) and scalar DM (bottom panel).

highly restricted by direct search (recall figure 5). We can also see that $m_{\chi_{2}}=2 \mathrm{TeV}$ closely mimic $Y_{2}=0$ case for fermion DM, while it does not completely do so for $\Omega_{S}$. This is because of very small annihilation cross-section of the scalar DM to SM compared to DM-DM conversion due to the choice of small $\lambda_{S H}$.

Let us now turn to direct search constraints of this two component DM set up. Feynman graphs for direct search contribution of the DM components are shown in figure 9 . Fermion DM $\left(N_{1}\right)$ has both $Z$ and Higgs mediated interaction, while the scalar $(S)$ interacts only through Higgs mediation. Direct search cross-sections for individual components are well known; however in two-component set up, the direct search cross-section for each 
component is folded by their fraction of relic density as: ${ }^{2}$

$$
\sigma_{\text {eff }}^{S I}(S)=\left(\frac{\Omega_{S} h^{2}}{\Omega_{T} h^{2}}\right) \sigma_{S}^{S I},
$$

and

$$
\sigma_{\text {eff }}^{S I}\left(N_{1}\right)=\left(\frac{\Omega_{N_{1}} h^{2}}{\Omega_{T} h^{2}}\right) \sigma_{N_{1}}^{S I}
$$

Spin independent direct search cross-sections for both DM components $\left(\sigma_{S}^{S I}\right.$ and $\left.\sigma_{N_{1}}^{S I}\right)$ are obtained from inserting the model into the code MicrOmegas, Belanger-ml-2014vza. No signal for DM in direct search experiments like LUX [9], XENON 1T [10] so far put a strong constraint on the WIMP-like DM scenarios as we have here. Recall that scalar DM lives only in the high mass region $(\geq 900 \mathrm{GeV})$ except for resonance $\left(\sim m_{h} / 2\right)$ and fermion DM lives in $\sin \theta \leq 0.1$ region with a small $\Delta m$ in their single component set up. The question is how much the above conclusions get relaxed in a two component set up with large DM-DM conversion as adopted here.

Case I: feeble DM-DM interactions with $\boldsymbol{Y}_{\mathbf{2}}=\mathbf{0}$. Let us now turn to relic density $\left(0.1133 \leq \Omega_{T} h^{2} \leq 0.1189\right)$ and SI direct search allowed parameter space of this two component model. We will first study the case for negligible DM-DM interactions with $Y_{2}=0$. The results are summarised in figure 10 . We show the relic density allowed parameter space in upper panel, in the left for $N_{1}$ and in the right for $S$. With $Y_{2}=0$, the two DM-components behave as if they are decoupled and the allowed parameter space only opens up in the under-abundant regions of those individual DMs (compare the single component cases as demonstrated before in figure 3 and figure 5). Different colour codes indicate the percentage of the individual DM density as indicated in the figure inset. It is understood that given a certain percentage of one DM, rest of DM relic density is composed of the other component. So any combination is essentially possible by relic density constraint. In the bottom panel of figure 10, we show the allowed parameter space after direct search constraints from PANDA where both DMs simaltaneously satisfy direct seach bound from PANDA [12]. Note here that there are no parameter space where effective DD cross-section (in eqs. (4.8), (4.9)) of both $N_{1}$ and $S$ DM simaltaneously goes beyond recent XENON-1T limit [11]. For fermion DM, direct search allowed parameter space spans the whole of under-abundant parameter space as it doesn't constrain the small $\Delta m$ region further with small singlet-doublet mixing $\sin \theta \leq 0.05$, as we have chosen for the scan. We have already explained that for fermion DM, direct search crucially controls $\sin \theta$ only, which is well below the required cut-off. On the other hand, scalar DM is severely constrained by direct search constraint in $m_{S}-\lambda_{S H}$ plane, which leaves Higgs resonance (not shown in the plot) and heavy Scalar DM mass region $\left(m_{S} \geq 900 \mathrm{GeV}\right)$ only. In the heavy scalar mass region, the relic density is $\gtrsim 80 \%$, therefore allowing only a tiny fraction of fermion DM. The whole analysis at $Y_{2}=0$ also shows that the presence of $s$-channel Higgs mediated DM-DM interactions to be very feeble to alter the freeze-out of any of the DM component as mentioned earlier.

\footnotetext{
${ }^{2}$ The actual limit from direct search on multipartite DM scenarios need to account for mass sensitivity on the nuclear recoil, the details can be found here [20,25].
} 

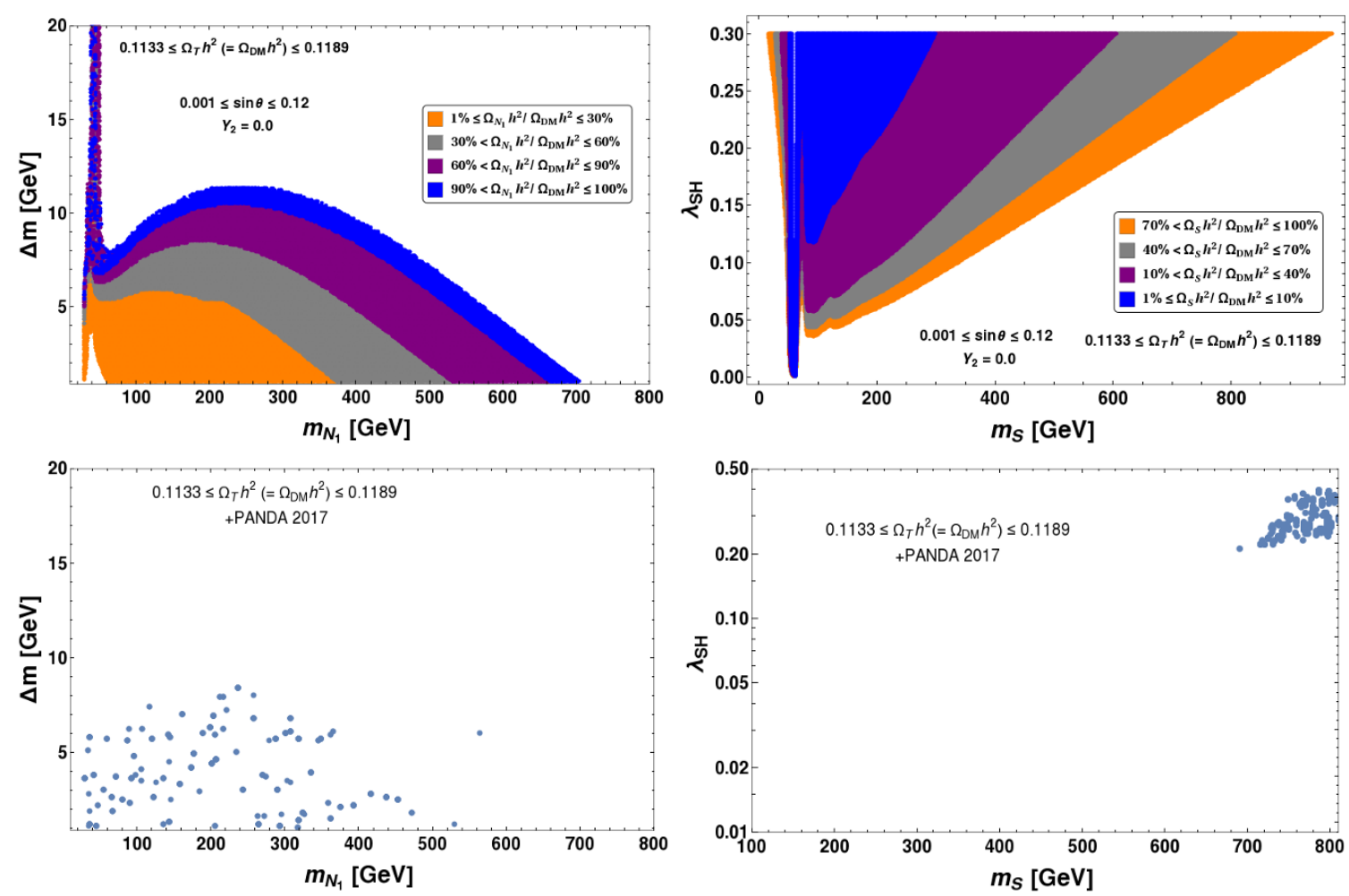

Figure 10. [Top Panel] Relic density allowed regions of two component DM scenario $\left\{N_{1}, S\right\}$ in $m_{N_{1}}-\Delta m$ (top left) and $m_{S}-\lambda_{S H}$ (top right) for $Y_{2}=0$. Different colour codes indicate the fraction of individual relic density $\frac{\Omega_{i}}{\Omega_{T}}$ varied in different ranges as mentioned in inset. [Bottom Panel] Relic density and direct detection (PANDA 2017 [12]) allowed parameter space in $m_{N_{1}}-\Delta m$ (bottom left) and $m_{S}-\lambda_{S H}$ (bottom right) planes.

Case II: the case of DM-DM interactions with $\boldsymbol{Y}_{2} \neq 0$. In figure 11, we show the relic density and direct search allowed parameter space of the model with a non-zero Yukawa coupling $\left(Y_{2} \neq 0,0.1 \leq Y_{2} \leq 1.6\right)$. Relic density allowed parameter space is shown in the upper panel for $N_{1}$ (in $m_{N_{1}}-\Delta m$ plane) on left and for $S$ (in $m_{S}-\lambda_{S H}$ plane) on right. Both possible mass hierarchies are studied and depicted; (i) $m_{N_{1}}>m_{S}$ by orange and (ii) $m_{S}>m_{N_{1}}$ by blue points. We see that when $m_{S}>m_{N_{1}}$, the whole $m_{S}-\lambda_{S H}$ parameter space is allowed (blue points in top right plot), where smaller $\lambda_{S H}$ is substituted by larger $Y_{2}$ appropriately. On the other hand, $N_{1}$ DM has the fate of single component DM with under abundance adjusted to the other component when $m_{S}>m_{N_{1}}$ (blue points in top left plot). This is exactly the other way round, when we choose $m_{S}<m_{N_{1}}$; the whole $m_{N_{1}}-\Delta m$ plane becomes allowed (orange points in top left plot) and $S$ has the fate of single component DM filling the under abundance region (orange points in top right plot). This is possible because of DM-DM conversion that we introduced in this model through the heavy mediator $\chi_{2}$ with $Y_{2}$ Yukawa interaction. With $m_{N_{1}}>m_{S}$, the effective annihilation required for fermion DM to acquire required relic density $\left(\Omega_{N_{1}} h^{2}<\Omega_{D M} h^{2}\right)$ in small $\sin \theta$ region no longer depends on small $\Delta m$ through co-annihilation because of additional annihilation channel to scalar DM. We will focus on this particular case 

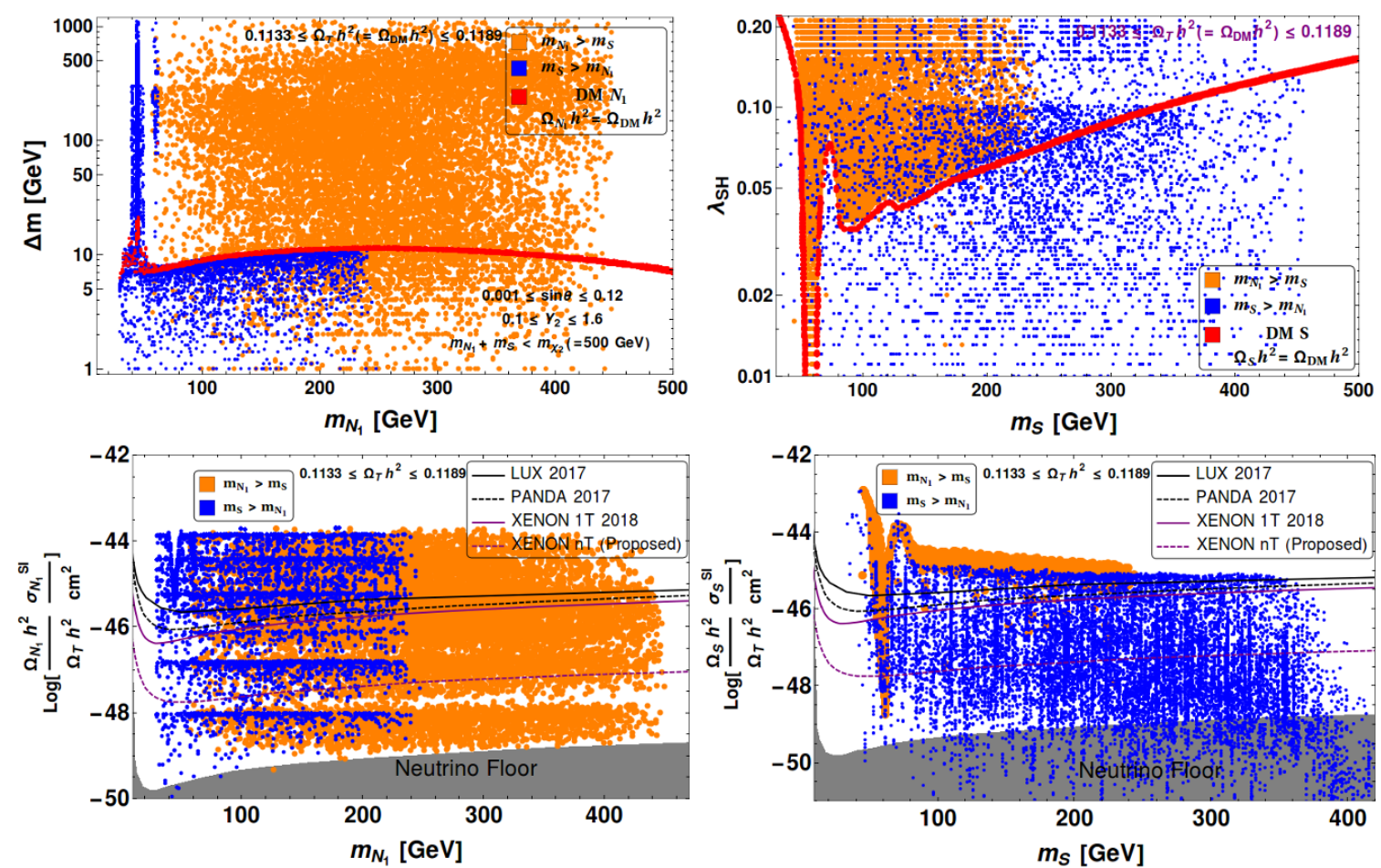

Figure 11. [Top Panel] Relic density allowed parameter space for two component DM model in $m_{N_{1}}-\Delta m$ (top left) and $m_{S}-\lambda_{S H}$ (top right) planes. Two mass hierarchies: $m_{N_{1}}>m_{S}$ (orange points) and $m_{S}>m_{N_{1}}$ (blue points) are shown in both plots. Red points depict the case of single component DM scenarios, for $N_{1}$ on the left and for $S$ on the right panel. [Bottom Panel] Relic density allowed points are shown in DM mass vs effective SI DM-nucleon cross-section planes; $\left(\frac{\Omega_{N_{1}} h^{2}}{\Omega_{T} h^{2}}\right) \sigma_{N_{1}}^{S I}-m_{N_{1}}$ in bottom left and $\left(\frac{\Omega_{S} h^{2}}{\Omega_{T} h^{2}}\right) \sigma_{S}^{S I}-m_{S}$ in bottom right. Limits from different DD experiments, LUX [9](black solid line), recent PANDA [12] (black dashed), XENON 1T [10] (purple solid line) and predicted XENON nT [11] (purple dotted line) are also indicated in the figures. Shadded region correspond to Neutrino floor where DM signal cannot be distinguish from neutrino background.

for collider signatures of this model at the LHC. In the bottom panel of figure 11, we show the effective SI direct search cross-section for both DM components at relic density allowed points $\left(\Omega_{i} h^{2}<\Omega_{D M} h^{2}\right)$ for both the mass hierarchies. The limits from LUX [9], PANDA [12], XENON 1T [10] and XENON nT [11] are shown. The plots in the bottom panel point out to a larger available parameter space for the heavier DM component. This is simply due to freeze-out of the heavier component being governed by DM-DM conversion, not affecting direct search significantly. The scans in figure 11 are limited to DM mass within $\lesssim 500 \mathrm{GeV}$ as it has been done for a mediator mass $m_{\chi_{2}}=500 \mathrm{GeV}$ to satisfy $m_{N_{1}}+m_{S}<m_{\chi_{2}}$.

The outcome of relic density and direct search (XENON 1T limits [10] from the bottom panel of figure 11) constraints put together yield figure 12. The constraints on fermion DM in $m_{N_{1}}-\Delta m$ plane (left) is obviously less restrictive as we choose small $\sin \theta \lesssim 0.05$ for the scan, thus allowing the whole parameter space with upto $\Delta m \gtrsim 500$ or more for $m_{N_{1}}>m_{S}$ 

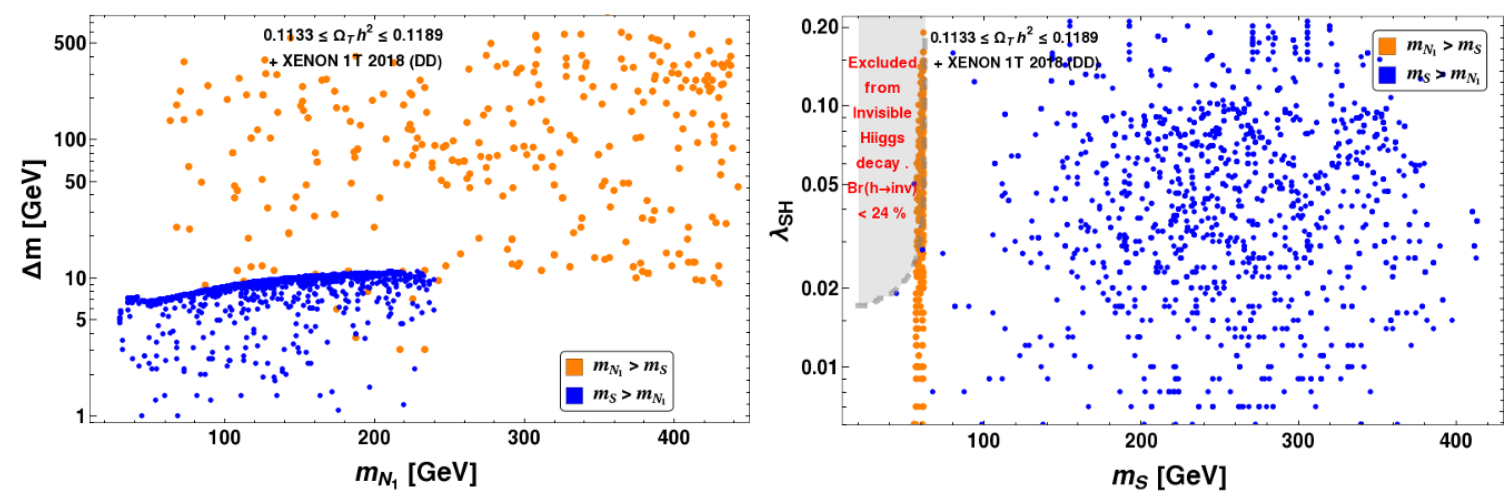

Figure 12. Relic density and direct search (XENON 1T data [10]) allowed parameter space is shown for both $N_{1}$ and $S$ components in $m_{N_{1}}-\Delta m$ (left) and $m_{S}-\lambda_{S H}$ (right) plane. Two possible mass hierarchies: $m_{N_{1}}>m_{S}$ (orange points) and $m_{S}>m_{N_{1}}$ (blue points) are indicated in both planes. Invisible Higgs branching constraint is also shown in the right panel which discards a significant part of $m_{S}<m_{h} / 2$ region.

(orange points in left plot) thanks to conversion to the scalar DM. This feature serves as the most interesting phenomenological outcome of this model, as we discuss in collider section. For $m_{N_{1}}>m_{S}$, the scalar DM however is allowed only in the resonance region $\left(m_{S} \sim m_{h} / 2\right)$ as can be seen by orange points in the right plot of figure 12 . This is already expected as direct search tames down the relic density allowed scalar DM parameter space absent DM-DM conversion. For the reverse hierarchy $m_{N_{1}}<m_{S}$ (blue points), fermion DM is allowed only in the under-abundant regions of its single component manifestation, whereas it allows a larger mass range of scalar DM, thanks again to the possible DM-DM conversion with a lighter $N_{1}$. Invisible Higgs branching ratio $\operatorname{Br}(h \rightarrow$ inv $)<0.24$ [42], puts a significant constraint for the scalar DM with $m_{S}<m_{h} / 2$. But for fermion DM, this doesn't discard any parameter space given the small values of $\sin \theta$ chosen for the scan.

A possible mass correlation of these two DM components is studied next and depicted in figure 13 in $m_{N_{1}}-m_{S}$ plane for satisfying relic density and direct search constraints. On the left panel, we show that the whole triangle designated by the kinematic limit $m_{N_{1}}+m_{S}<m_{\chi_{2}}$, with $m_{\chi_{2}}=500 \mathrm{GeV}$ chosen for the scan is allowed by relic density constraint. However direct search (XENON1T data [10]) restricts it significantly for $m_{N_{1}}>$ $m_{S}$, allowing only scalar DM to lie in resonance $m_{h} / 2$, while it is not that restrictive for the other hierarchy $m_{N_{1}}<m_{S}$, as shown by the spread of blue points filling almost entirely the upper part of the triangle. The thick black line depicting $m_{N_{1}}=m_{S}$ separates these two heirarchies. The plot on the right panel shows the allowed points in $m_{N_{1}}-m_{S}$ plane to satisfy relic density for different ranges of $\Delta m$. It shows that small $1 \leq \Delta m \leq 12 \mathrm{GeV}$ is allowed throughout the parameter space while large $\Delta m$ is restricted to $m_{N_{1}}>m_{S}$ as we already discussed. For $m_{N_{1}}<m_{S}$, one can have larger $\Delta m$ allowed only in the resonance region $m_{N_{1}} \sim m_{h} / 2$ and $\sim m_{Z} / 2$.

Another important question is to know the percentage of fermion or scalar DM component present in the allowed parameter space of this two component model. We show the outcome of this exercise in figure 14, for fermion DM in $m_{N_{1}}-\Delta m$ plane. The other com- 

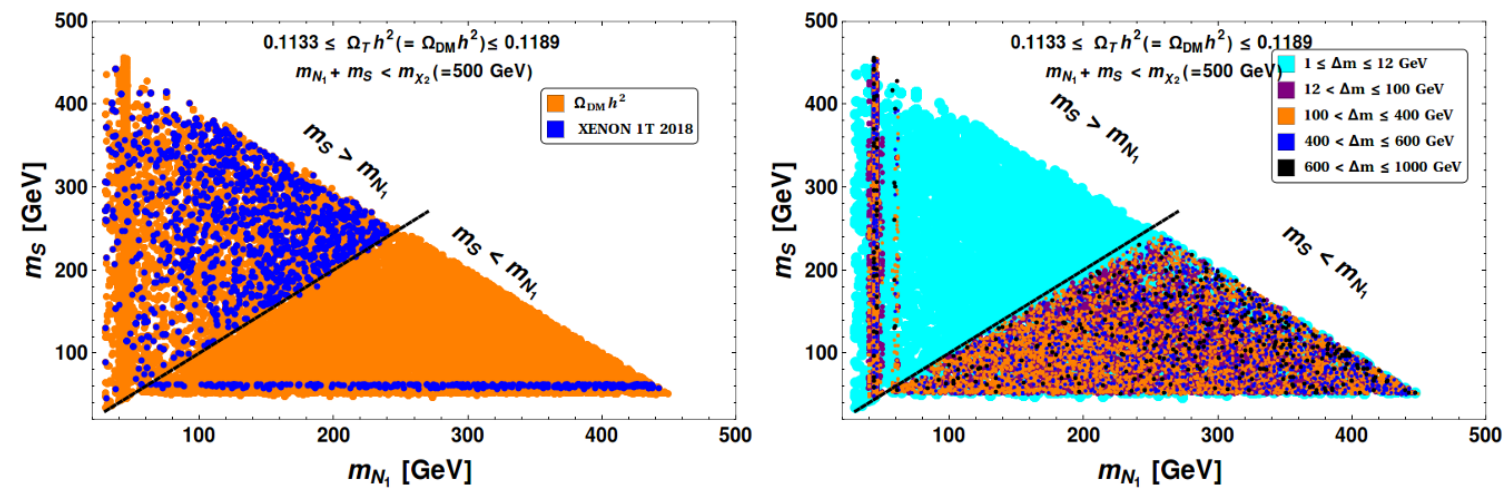

Figure 13. Mass correlation of the two DM components in $m_{N_{1}}-m_{S}$ plane. [Left Panel] Relic density allowed parameter space is shown by orange points and direct search constraint from XENON 1T [10] on both $N_{1}$ and $S$ is shown by blue points. Black solid line corresponding to $m_{N_{1}}=m_{S}$ segregates the two possible hierarchies: the one above corresponds to $m_{S}>m_{N_{1}}$ and the region below has $m_{S}<m_{N_{1}}$. [Right Panel] Relic density allowed points for different ranges of $\Delta m$ shown with different colour codes.
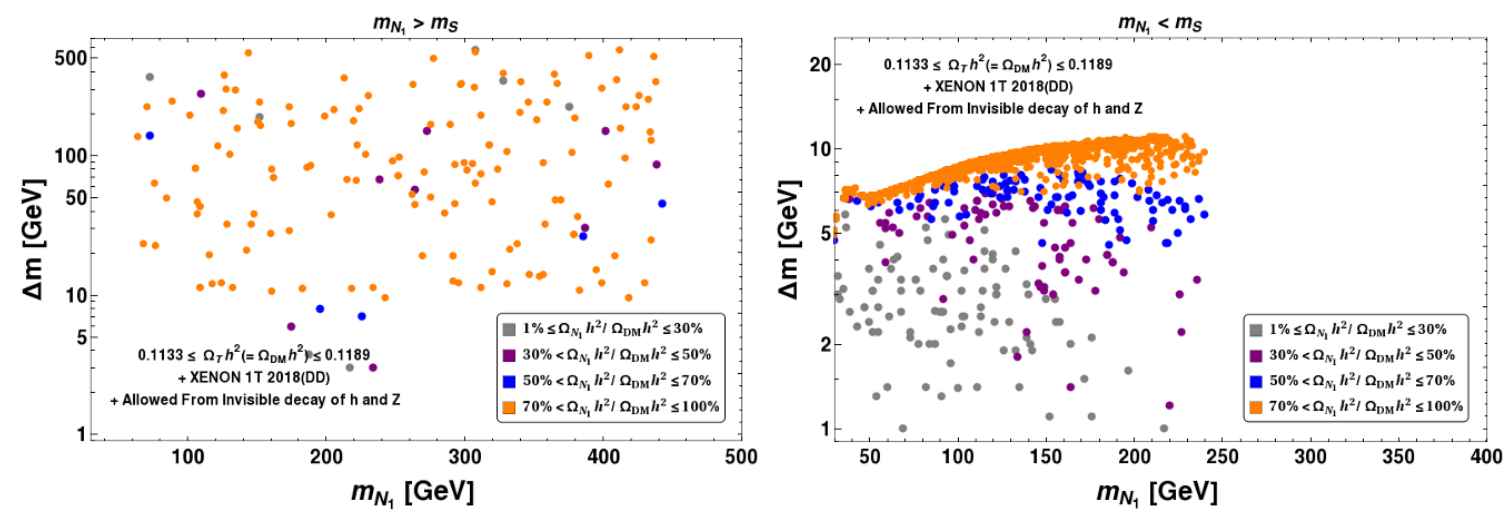

Figure 14. Relic density, direct search (XENON 1T [10]) and invisible decay constrain of Higgs and $Z$ boson [42] allowed parameter space of the two component model (with $Y_{2} \neq 0$ ) in $m_{N_{1}}-\Delta m$ plane. Percentage of fermion DM component in total relic density within different ranges are shown by different coloured points as detailed in the figure inset. Two different hierarchies $m_{S}<m_{N_{1}}$ and $m_{S}>m_{N_{1}}$ are shown separately in left and right panel respectively.

ponent $(S)$ just fills the rest of it and can be gauged from this figure itself. Two possible mass hierarchies $m_{S}<m_{N_{1}}$ and $m_{S}>m_{N_{1}}$ are shown separately in left and right panel respectively. Fermion DM content in total relic density (for different ranges in percentage) is shown by different colour codes mentioned in the figure inset. All the points also additionally satisfy direct search constraint from XENON1T data [10] and invisible decay constraint of Higgs and $Z$ [42]. The bottom line is that for $m_{N_{1}}>m_{S}$, the larger share of DM density is carried by fermion DM with $\Delta m \gg 12 \mathrm{GeV}$ as it becomes enough to bring the annihilation in the right ballpark through conversion to the scalar DM component (with small $\sin \theta$ ), while the scalar DM anyway has a large annihilation cross section (and therefore smaller relic density) as it requires to be in the Higgs resonance region $\left(m_{S} \sim m_{h} / 2\right)$ to 

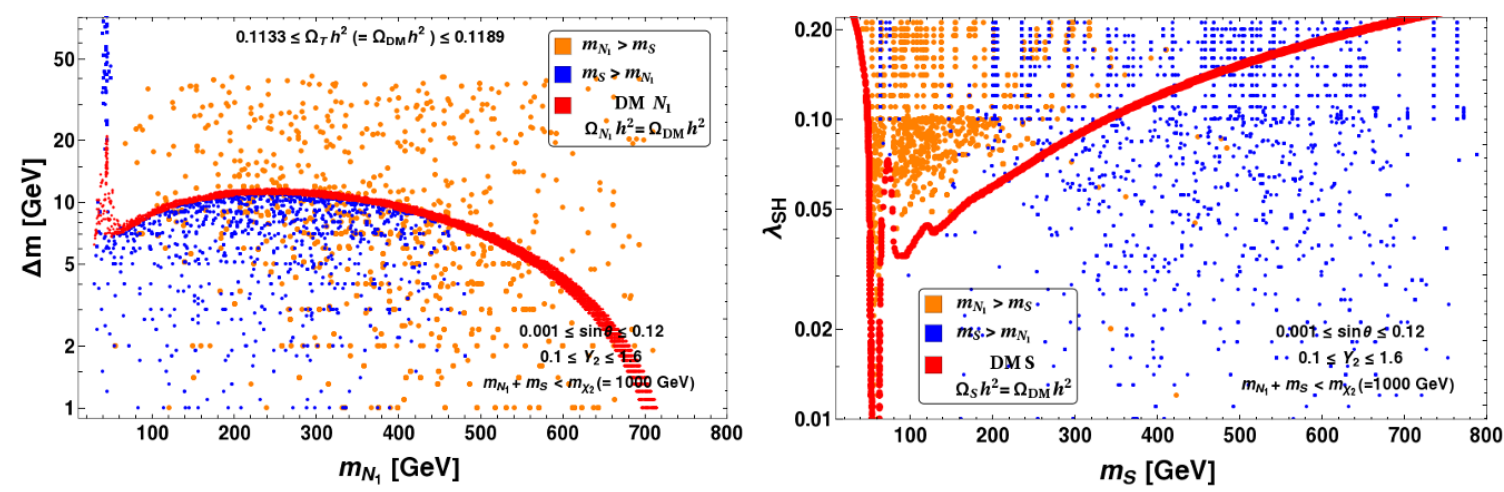

Figure 15. Total relic density allowed regions of two component DM model in $m_{N_{1}}-\Delta m$ (left panel) and $m_{S}-\lambda_{S H}$ plane (right panel) with $Y_{2} \neq 0$ for mediator mass $m_{\chi_{2}}=1000 \mathrm{GeV}$. Two mass hierarchies are shown in different colour codes: $m_{N_{1}}>m_{S}$ (orange points) and $m_{S}>m_{N_{1}}$ (blue points). Red points correspond to the case of single component DM scenarios for $N_{1}$ on left and for $S$ on right panel.

address direct search bound. For the other hierarchy $m_{N_{1}}<m_{S}$, under abundant regions of the single component fermion DM is filled up with different percentage as the scalar DM has the freedom to adjust its relic density through its annihilation to SM plus fermion DM.

So far we have discussed the allowed DM parameters space for the model with a moderate choice of mediator mass, $m_{\chi_{2}}=500 \mathrm{GeV}$. Now we choose a higher value of $\chi_{2}$ mass, $m_{\chi_{2}}=1000 \mathrm{GeV}$ to depict relic density allowed limit in $m_{N_{1}}-\Delta m$ plane (left) and $m_{S}-\lambda_{S H}$ plane (right) of figure 15. Allowed parameter space in $m_{N_{1}}-\Delta m$ plane becomes more restrictive ( $\Delta m$ spanning roughly upto $\sim 50 \mathrm{GeV}$ compared to $500 \mathrm{GeV}$ with $m_{\chi_{2}}=500 \mathrm{GeV}$ ) even with $m_{N_{1}}>m_{S}$ due to suppressed t-channel DM-DM conversion $\overline{N_{i}} N_{j} \rightarrow S S$ due to the heavy mediator $\left(m_{\chi_{2}}\right)$. Comparatively, larger parameter space is available for scalar DM $S$ as shown in right panel of figure 15 in $m_{S}-\lambda_{S H}$ plane. This is possible as $S S \rightarrow \overline{N_{i}} N_{j}$ with $\left(Y_{2} \neq 0.0\right)$ still dominate over scalar DM annihilation to SM (controlled by portal coupling $\lambda_{S H}$ ) even with a heavy mediator mass. This feature has already been pointed out while discussing the outcome of DM-DM conversion cross-sections in figure 8.

Finally, to summarise the main outcome of the DM analysis is to see that heavier DM component enjoys annihilation to lighter DM for thermal freeze out, relaxing its interaction to visible sector and thus reducing the constraints from direct search cross-sections. Specifically for the two-component case, when scalar DM is heavier than the fermion DM, the Higgs portal coupling can be reduced significantly allowing the scalar DM to be allowed through the entire DM mass plane. On the other hand, when the fermion DM is heavier than the scalar DM, it relaxes the mass difference with the charge companion, allowing larger $\Delta m$. No relaxation is possible however for $\sin \theta$ as larger values of mixing is still discarded by $Z$ mediated direct search. The relaxation of $\Delta m$ plays a crucial role in achieving collider signatures of fermion DM as we illustrate next. We choose a set of benchmark points allowed by relic density and direct search in table 2 for performing collider analysis, where above features are apparent. 


\begin{tabular}{|l|c|c|c|c|c|c|}
\hline BPs & $\left\{m_{N_{1}}, m_{S}, \lambda_{S H}, Y_{2}, \sin \theta\right\}$ & $\Delta m$ & $\Omega_{N_{1}} h^{2}$ & $\Omega_{S} h^{2}$ & $\begin{array}{c}\left(\frac{\Omega_{N_{1}} h^{2}}{\Omega_{D M} h^{2}}\right) \sigma_{N_{1}}^{S I} \\
\left.\text { (in } \mathrm{cm}^{2}\right)\end{array}$ & $\begin{array}{c}\left(\frac{\Omega_{S} h^{2}}{\Omega_{D M} h^{2}}\right) \sigma_{S}^{S I} \\
\left.\text { in } \mathrm{cm}^{2}\right)\end{array}$ \\
\hline BPA1 & $\{79,256,0.029,0.2,0.02\}$ & 6.1 & 0.0546 & 0.0641 & $6.8 \times 10^{-48}$ & $5.9 \times 10^{-47}$ \\
\hline BPA2 & $\{276,58,0.010,0.9,0.02\}$ & 50 & 0.1092 & 0.0054 & $1.5 \times 10^{-48}$ & $1.5 \times 10^{-47}$ \\
\hline BPA3 & $\{131,61,0.026,0.9,0.01\}$ & 101 & 0.1171 & 0.0012 & $1.0 \times 10^{-48}$ & $1.5 \times 10^{-47}$ \\
\hline BPA4 & $\{102,62,0.010,0.9,0.02\}$ & 193 & 0.1144 & 0.0010 & $1.8 \times 10^{-47}$ & $2.0 \times 10^{-48}$ \\
\hline BPA5 & $\{135,58,0.004,1.0,0.02\}$ & 295 & 0.0840 & 0.0313 & $1.5 \times 10^{-47}$ & $1.0 \times 10^{-47}$ \\
\hline BPA6 & $\{127,62,0.020,0.9,0.01\}$ & 377 & 0.1136 & 0.0004 & $1.4 \times 10^{-48}$ & $3.0 \times 10^{-48}$ \\
\hline BPA7 & $\{144,62,0.032,0.9,0.02\}$ & 541 & 0.1152 & 0.0002 & $2.7 \times 10^{-47}$ & $4.2 \times 10^{-48}$ \\
\hline
\end{tabular}

Table 2. Some benchmark points allowed by relic density, direct search and invisible Higgs and $Z$ decay limit for mediator mass, $m_{\chi_{2}}=500 \mathrm{GeV}$. DM masses, couplings, relic density of individual components and effective SI direct search cross-sections are mentioned. All the masses are in GeVs. We mainly focus on $m_{N_{1}}>m_{S}$ excepting for BPA1.

\section{Two component DM in presence of additional heavy scalar}

In the two component DM set up, lighter DM component behaves almost as a single component candidate, due to the absence of additional channels for annihilation, thus occupying only under abundant regions accessible from relic density. For $m_{N_{1}}>m_{S}$, a large mass splitting $\Delta m$ can be achieved for a moderated value of mediation mass $m_{\chi_{2}} \sim$ $500 \mathrm{GeV}$. But at the same time, the scalar DM can only be accommodated at the resonance region, $m_{S} \approx \frac{m_{H}}{2}$ (see figure 12 and table 2 ). This is predictive and restrictive at the same time. This situation however alters significantly if the scalar sector is enlarged with a heavy real scalar $S_{H}$ which has same charge like $S$ under $\mathcal{Z}_{2} \times \mathcal{Z}_{2}^{\prime}$ as: $S[-,-]$ and $\mathrm{S}_{\mathrm{H}}[-,-][21,31]$. We briefly discuss such a possibility here. The relevant interacting scalar potential is given by:

$$
V\left(S, S_{H}\right) \supset \frac{1}{2} m_{S}^{2} S^{2}+\frac{1}{2} m_{S_{H}}^{2} S_{H}^{2}+\frac{\lambda_{S H}}{2}\left(H^{\dagger} H-\frac{v^{2}}{2}\right)\left(S^{2}+S_{H}^{2}\right)+\lambda_{C H}\left(H^{\dagger} H-\frac{v^{2}}{2}\right) S S_{H},
$$

where $m_{S_{H}}$ is the heavy scalar mass and $\lambda_{C H}$ is additional (co-annihilation type) Higgs portal coupling. Due to the presence of this interaction, $\lambda_{C H}\left(H^{\dagger} H\right) S S_{H}$, a new coannihilation channel, $S S_{H} \rightarrow$ SM SM opens up. $S_{H}$ having same charge as of $S$, is not stable and therefore is not a DM. But the possibility of co-annihilation provides additional channel for scalar DM to freeze out, while it does not contribute to direct search. This is similar to the co-annihilation processes already present in the fermion DM sector. With this, even for $m_{N_{1}}>m_{S}$, the scalar DM can be allowed in a large parameter space beyond resonance. Presence of this heavy scalar, also augments dark sector Yukawa interaction providing additional contribution to DM-DM conversion:

$$
\mathcal{L}_{\mathcal{D} \mathcal{M}}{ }^{Y u k}=-Y_{2}\left(\overline{\chi_{1}} \chi_{2} S+\text { h.c. }\right)-Y_{2}^{\prime}\left(\overline{\chi_{1}} \chi_{2} S_{H}+\text { h.c. }\right) .
$$

In our numerical analysis, we assume $Y_{2}=Y_{2}^{\prime}$ for simplicity. 

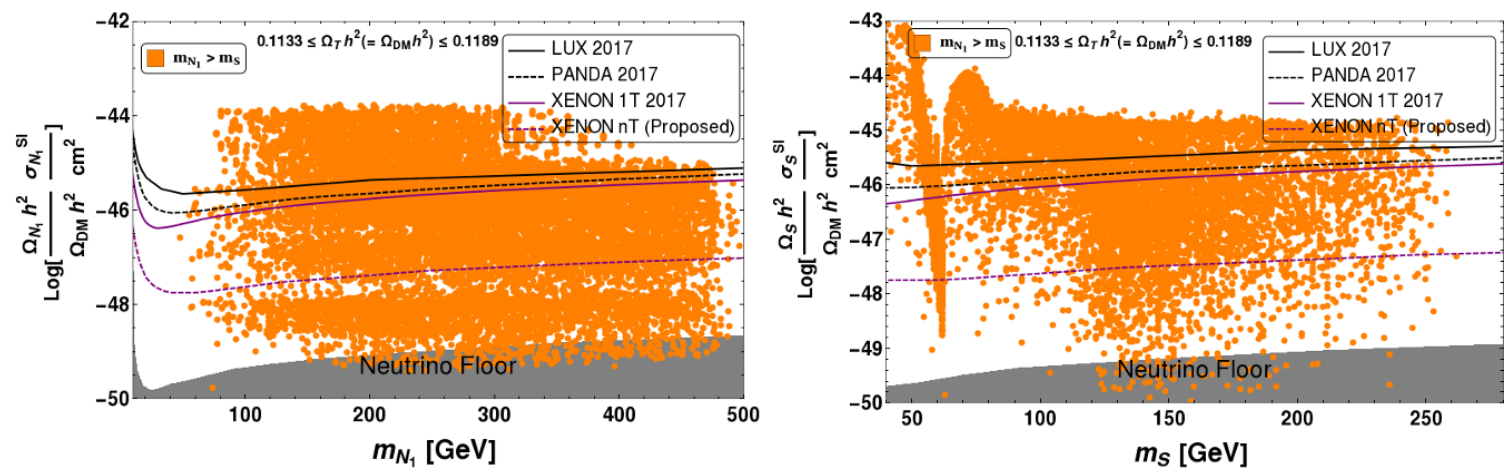

Figure 16. Relic density allowed points plotted in DM mass vs effective SI DM-nucleon crosssection plane: $\left(\frac{\Omega_{N_{1}} h^{2}}{\Omega_{D M} h^{2}}\right) \sigma_{N_{1}}^{S I}-m_{N_{1}}$ plane (left) and $\left(\frac{\Omega_{S} h^{2}}{\Omega_{D} h^{2}}\right) \sigma_{S}^{S I}-m_{S}$ plane (right) in presence of heavy scalar $S_{H}$. Upper bounds on SI DM-nucleon cross-section from LUX [9] (black solid line), recent PANDA [12] (black dashed), XENON 1T [10] (purple solid line) and predicted XENON $\mathrm{nT}$ [11] (purple dotted line) are also indicated in the figures. We have chosen the mass hierarchy: $m_{N_{1}}>m_{S}$ and the mediator mass $m_{\chi_{2}}=500 \mathrm{GeV}$.
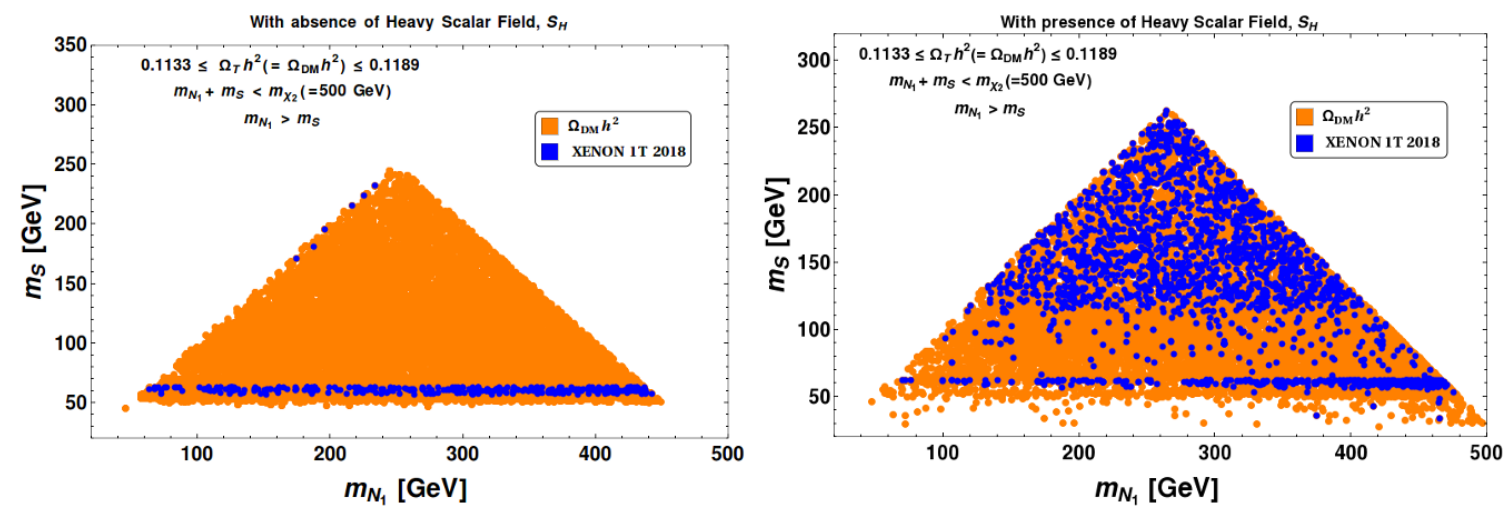

Figure 17. Allowed region of parameters space in $m_{N_{1}}-m_{S}$ plane, which satisfy relic density (Orange points) and direct search constraints for both $N_{1}$ and $S$ by XENON1T data (blue points) for $m_{N_{1}}>m_{S}$. In left panel, we show the original two component scenario in absence of heavy scalar $\left(S_{H}\right)$ and in the right panel, we show it in presence of additional heavy scalar $S_{H}$.

The first outcome of this extended two component framework is to show a large parameter space available to the scalar DM through relic density and direct search bounds with the hierarchy $m_{N_{1}}>m_{S}$. This is illustrated in figure 16. The direct search crosssection for fermion DM in relic density allowed points is shown on the left plot, while that for the scalar is shown in the right panel. We see in the right plot that orange points now span allover the plane with a huge number below the direct search limit unlike being only available in resonance region with the previous case (compare bottom right plot of figure 11).

A mass correlation for two DM components is shown in figure 17 and compared between the original framework (left) to that in presence of an additional heavy scalar (right). We show that with $m_{N_{1}}>m_{S}$, presence of co-annihilation in the scalar sector allows the scalar 

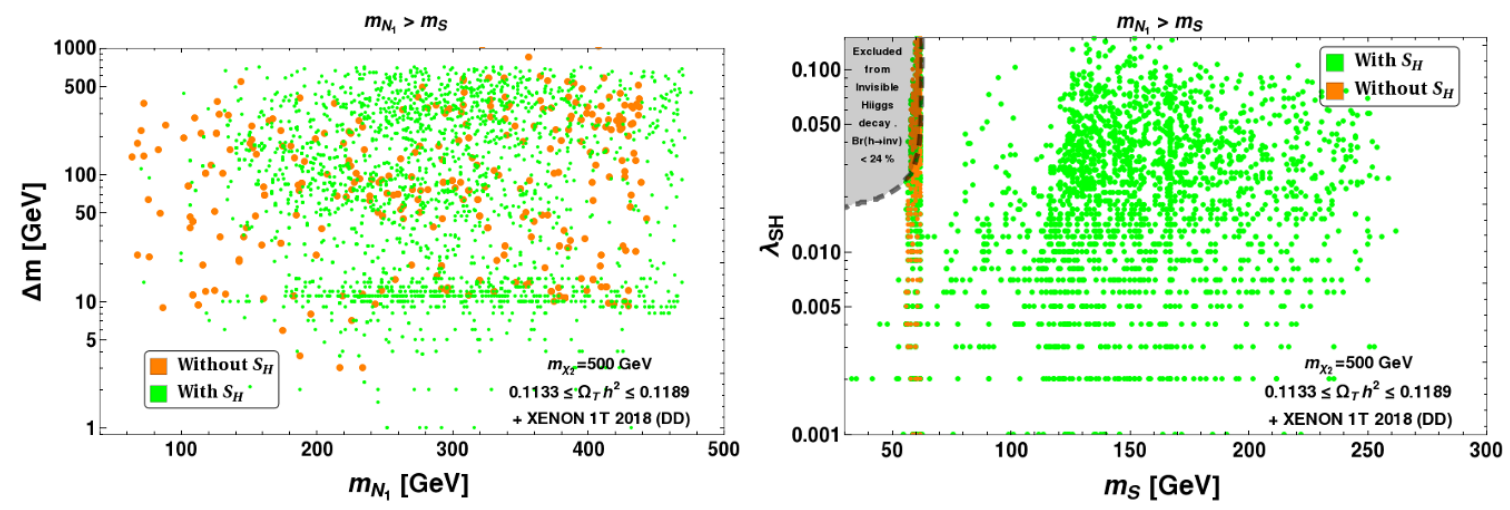

Figure 18. Relic density and direct search (XENON 1T [10]) allowed parameter space of the two component model compared between two cases: (i) original model, in absence of the heavy scalar $\left(S_{H}\right)$ (orange points) and (ii) in presence of a heavy scalar $\left(S_{H}\right)$ (green points) in $m_{N_{1}}-\Delta m$ plane (left) and $m_{S}-\lambda_{S H}$ plane (right).

\begin{tabular}{|l|c|c|c|c|c|c|}
\hline BPs & $\left\{m_{N_{1}}, m_{S}, m_{S_{H}}, \lambda_{S H}, \lambda_{C H}, Y_{2}, \sin \theta\right\}$ & $\Delta m$ & $\Omega_{N_{1}} h^{2}$ & $\Omega_{S} h^{2}$ & $\begin{array}{c}\left(\frac{\Omega_{N_{1}} h^{2}}{\Omega_{D M} h^{2}}\right) \sigma_{N_{1}}^{S I} \\
\left.\text { (in } \mathrm{cm}^{2}\right)\end{array}$ & $\begin{array}{c}\left(\frac{\Omega_{S} h^{2}}{\Omega_{D M} h^{2}}\right. \\
\left.\text { (in } \mathrm{cm}^{2}\right)\end{array}$ \\
\hline BPB1 & $\{200,150,205,0.006,0.8,0.7,0.01\}$ & 50 & 0.1144 & 0.0030 & $9.7 \times 10^{-49}$ & $3.3 \times 10^{-49}$ \\
\hline BPB2 & $\{202,118,131,0.002,0.3,0.9,0.04\}$ & 101 & 0.0303 & 0.0838 & $7.2 \times 10^{-47}$ & $1.5 \times 10^{-48}$ \\
\hline BPB3 & $\{183,113,135,0.009,0.8,0.8,0.04\}$ & 201 & 0.0462 & 0.0680 & $1.2 \times 10^{-46}$ & $3.0 \times 10^{-47}$ \\
\hline BPB4 & $\{310,153,203,0.052,0.6,0.7,0.02\}$ & 300 & 0.1112 & 0.0100 & $2.0 \times 10^{-47}$ & $5.8 \times 10^{-47}$ \\
\hline BPB5 & $\{424,91,109,0.004,0.6,1.1,0.03\}$ & 503 & 0.0238 & 0.0945 & $2.7 \times 10^{-47}$ & $1.1 \times 10^{-47}$ \\
\hline
\end{tabular}

Table 3. Benchmark points allowed by relic density, direct search and invisible Higgs decay limit in presence of a heavy scalar $S_{H}$. Input parameters (masses and couplings), relic densities of individual components and direct search cross-sections are mentioned. All the masses are in GeVs.

DM to be present in a larger parameter space after satisfying direct search constraints (plot on the right panel).

Finally, we compare relic density and direct search (XENON 1T [10]) allowed parameter space of the two component model in presence of $S_{H}$ (green points) to that in absence of $S_{H}$ (orange points) for both fermion DM and scalar DM components in figure 18. As expected, we see that for fermion DM, in $m_{N_{1}}-\Delta m$ plane (on left panel), there is no difference between these two cases, while for the scalar DM $S$, the presence of the heavy scalar $S_{H}$ allows almost all of the plotted parameter space (green points on the right panel) due to coannihilation. A few benchmark points are indicated in table 3 to show the effect of relaxing the case for scalar DM in presence of $S_{H}$ for $m_{N_{1}}>m_{S}$. They should be contrasted with those in table 2 .

\section{Collider searches at LHC}

Collider signature of this model includes searches for scalar and fermion DM. The scalar DM sector doesn't give any novel signature being comprised only of a singlet. Only possible signature can be the production of $S$ through Higgs portal coupling associated with initial 

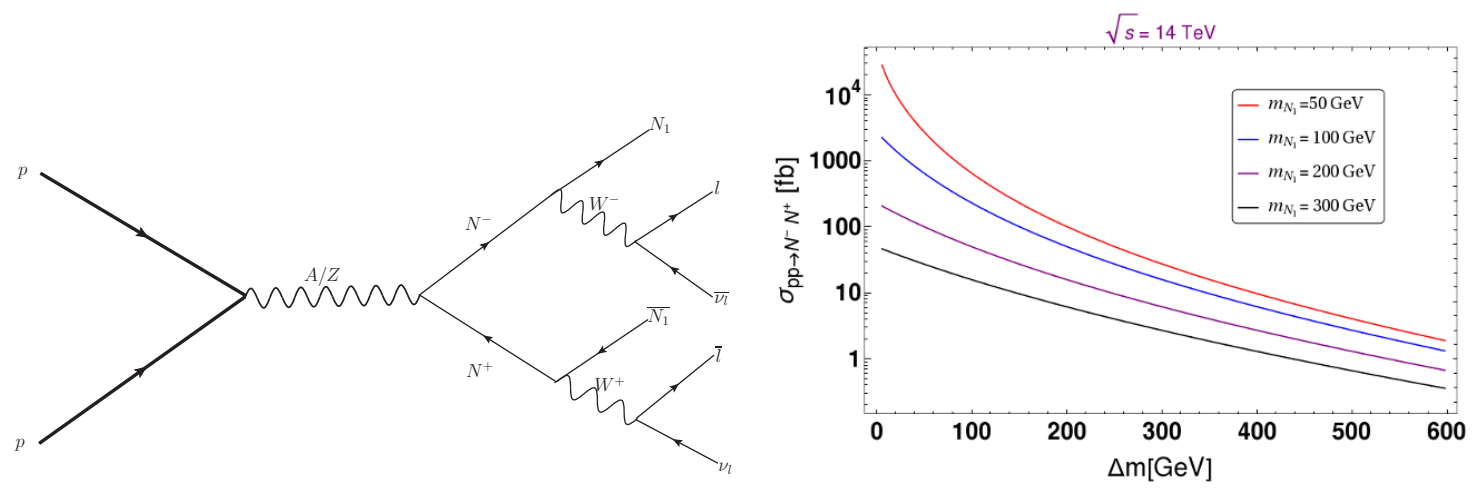

Figure 19. [Left] Feynman diagram for signal process $p p \rightarrow N^{+} N^{-}$, resulting in hadronically quiet opposite sign dilepton plus missing energy $\left(\ell^{+} \ell^{-}+\left(\mathbb{E}_{T}\right)\right)$ events. [Right] Variation in production cross section, $\sigma_{p p \rightarrow N^{+} N^{-}}$with $\Delta m\left(=m_{N^{ \pm}}-m_{N_{1}}\right)$ for different values of DM mass $m_{N_{1}}$ [mentioned at figure inset] for centre-of-mass energy $\sqrt{s}=14 \mathrm{TeV}$ at LHC.

state radiation (ISR), yielding mono jet/mono-X signal (higher jet multiplicity can occur suppressed by further jet radiation) plus missing energy [45]. Given the limit on the Higgs portal coupling $\left(\lambda_{S H}\right)$ and DM mass set by the relic density and direct search bound of the model, even in the two component set up, the signal cross-section is very weak to probe anything at near future run of LHC given a huge SM background for such final states. ${ }^{3}$ On the other hand, fermion DM consisting of an admixture of vector-like singlet and doublet leptons, has better prospect of getting unravelled at LHC. This is of particular interest due to the possibility of producing the charged companions of fermion doublet $\left(\mathrm{N}^{+} \mathrm{N}^{-}\right)$ at LHC. They eventually decay to DM with off/on- shell $\mathrm{W}$ mediation to leptonic final states to yield opposite sign dilepton plus missing energy as pointed out in the left side of figure 19. Therefore our interest lies in:

$$
\text { Signal }: \ell^{+} \ell^{-}+\left(\mathbb{E}_{\mathrm{T}}\right) \text {, }
$$

where $\ell$ includes electrons and muons. ${ }^{4}$ However, the detectability of such a signal depends on the effective reduction of corresponding SM background contribution. We will discuss below how the presence of a second (lighter) DM component as considered in this model framework, enhance the possibility of detecting such signals at LHC. Similar signal events appear for different other models, see for example [46, 47].

Signal strength is mainly dictated by the production cross-section for $p p \rightarrow N^{+} N^{-}$at LHC. This cross-section is essentially a function of $m_{N^{ \pm}}$and is independent of mixing angle $\sin \theta$. Therefore, one can recast the cross-section as a function of $\Delta m$ for a fixed DM mass (Given $m_{N^{ \pm}}=m_{N}+\Delta m$ ). This is shown in the right panel of figure 19 for some different fixed DM masses (mentioned in the figure inset) with centre-of-mass-energy $\sqrt{s}=14 \mathrm{TeV}$. Essentially, this is to show that production cross-section is a falling function of charged fermion masses, but, as $\Delta m$ plays a crucial role in further decay of the produced charged

\footnotetext{
${ }^{3}$ Even though the presence of a heavy scalar $S_{H}$ adds to the freedom of choosing a larger span of scalar DM mass, the strength of the cross-section still is determined by the small $\lambda_{S H}$.

${ }^{4}$ Tau detection is harder due to hadronic decay modes.
} 
fermions, we have chosen such parametrisation. We already elaborated that even in the two component set up, direct search crucially tames $\sin \theta \leq 0.1$, it is important to choose a process which is not suppressed by small mixing angle. Therefore, this is the only process of interest. However, also note that, we do not consider the production of heavy neutral fermion $N_{2}$ in this analysis (although some of the processes like $N^{ \pm} N_{2}$ are not suppressed by $\operatorname{small} \sin \theta)$, which decays through neutral current ( $Z$ mediation) interaction to DM $N_{1}$ with 100 percent branching ratio. But such signals will be completely washed out by the invariant mass-cut of the leptons not to lie within $Z$-mass window, that we must apply to suppress SM background (as explained shortly). There are two kinematic constraints that we obey for characteristic collider signal that we discuss here: (i) $m_{\chi_{2}}>m_{N_{1}}+m_{S}$ and (ii) $m_{N_{1}}>m_{S}$. The second constraint allows us to choose a large $\Delta m$ as explained earlier and plays an important role in separating the signal from SM background. Most of the benchmark points in table 2 and all in table 3 , follow the characteristics mentioned above. We will analyse signal strength for some such benchmark points. Although, we use benchmark points from table 2 here, they can also be thought as similar points (with same $\Delta m$ ) from table 3, where we have further relaxation on scalar DM mass (which do not play a role in the collider signature for fermion DM).

Before getting into the collider analysis, let us briefly explain the experimental environment of LHC, which mainly involves identification of leptons, jets and unclustered objects. Some important variables are also used in the analysis such as missing energy, invariant mass of the dilepton in the final state and scalar sum of the transverse momentum of all the visible objects in the final state. They are identified as follows:

- Lepton $(l=e, \mu)$ : leptons are identified with a minimum transverse momentum $p_{T}>20 \mathrm{GeV}$ and pseudorapidity $|\eta|<2.5$. Although the present sensitivity of the detector allows further soft leptons to be identified, we find that such a $p_{T}$ cut also helps to tame SM background. Leptons require to be isolated if their mutual distance in the $\eta-\phi$ plane is $\Delta R=\sqrt{(\Delta \eta)^{2}+(\Delta \phi)^{2}} \geq 0.2$, while the separation with a jet requires $\Delta R \geq 0.4$.

- Jets $(j)$ : jets are formed for simulated signal and background events using cone algorithm PYCELL inbuilt in Pythia event generator. All the partons within $\Delta R=0.4$ from the jet initiator cell are included to form the jets. We require $p_{T}>20 \mathrm{GeV}$ for a clustered object to be identified as jets in hadron calorimeter (HCAL). Jets are isolated from unclustered objects with $\Delta R>0.4$. Note here, that although jets are not present in the final state, we require a specific jet identification criteria to demand the final state has zero jets.

- Unclustered Objects: all the final state objects with low $p_{T}$, which are neither clustered to form jets, nor passes through the identification criteria to become isolated leptons, belong to such category. Hence all particles with $0.5<p_{T}<20 \mathrm{GeV}$ and $2.5<|\eta|<5$, are considered as unclustered objects. They only contribute to missing energy. 
- Missing Energy $\left(E_{T}^{\prime}\right)$ : the transverse momentum of all those electromagnetic charge neutral particles not registered in the detector, can be estimated form the momentum imbalance in the transverse direction associated to the visible particles. Thus missing energy (MET) is defined as:

$$
E_{T}^{\prime}=-\sqrt{\left(\sum_{\ell, j, u n c .} p_{x}\right)^{2}+\left(\sum_{\ell, j, u n c .} p_{y}\right)^{2}},
$$

where the sum runs over all visible objects that include the leptons and jets, and the unclustered components. Missing energy is the most significant variable to identify DM at collider.

- Effective Mass $\left(H_{T}\right)$ : effective mass of an event is identified here with the scalar sum of the transverse momentum of detectable objects in an event, namely lepton and jets as follows:

$$
H_{T}=\sum_{\ell, j}\left(p_{T}\right)_{\ell, j} .
$$

Effective mass usually also includes missing energy as a component added in the scalar sum. However, here we use $H_{T}$ without including $E_{T}^{\prime}$, as we will use $E_{T}^{\prime}$ as a separate variable in combination of $H_{T}$ cut anyway to segregate signal from SM background.

- Invariant mass $\left(m_{\ell \ell}\right)$ : invariant mass of opposite sign dilepton is an important variable to segregate SM background from the signal, as it hints to the parent particle mass from which the leptons have been produced. This is defined as:

$$
m_{\ell \ell}=\sqrt{\left(\sum_{\ell} p_{x}\right)^{2}+\left(\sum_{\ell} p_{y}\right)^{2}+\left(\sum_{\ell} p_{z}\right)^{2}} .
$$

We inserted the model in Feynrules [48] and passed to Madgraph [49] to generate signal events, which were further analysed in Pythia [50] to reconstruct leptons, jets and other variables discussed above. The dominant SM backgrounds have been generated in Madgraph [49] and then showered through Pythia [50]. We have identified dominant SM backgrounds for hadronically quiet opposite sign dilepton events as the production of: $t \bar{t}$, $W^{+} W^{-}, W^{ \pm} Z, Z Z, W^{+} W^{-} Z$ and Drell-Yan. We have also used appropriate $K$-factors to incorporate the Next-to-Leading order (NLO) cross section for the backgrounds. The $K$-factors chosen are as [51] for $t \bar{t}: K=1.47, W W: K=1.38, W Z: K=1.61$, $Z Z j: K=1.33$, Drell-Yan: $K=1.2$. We have used CTEQ 6L [52] parton distribution function and subprocess centre-of-mass-energy $(\sqrt{\hat{s}})$ as jet energy scale for the analysis.

Most important outcome of this analysis is summarised in figure 20, where the distribution of the signal events with respect to $\mathbb{E}_{T}, m_{\ell \ell}$ and $H_{T}$ are shown in top left, top right and bottom panel. We have chosen different $\Delta m$ (from the benchmark points as in table 2) upto as large as $\sim 500 \mathrm{GeV}$ allowed by relic density and direct search for illustration. In top left figure, we see that with larger $\Delta m$, missing energy distribution becomes flatter and the peak shifts to a higher value. When this is contrasted with the same distributions from those of SM background contributions as pointed out in figure 21, we see that the separation of signal events from those of the background becomes easier at high $\Delta m$. 

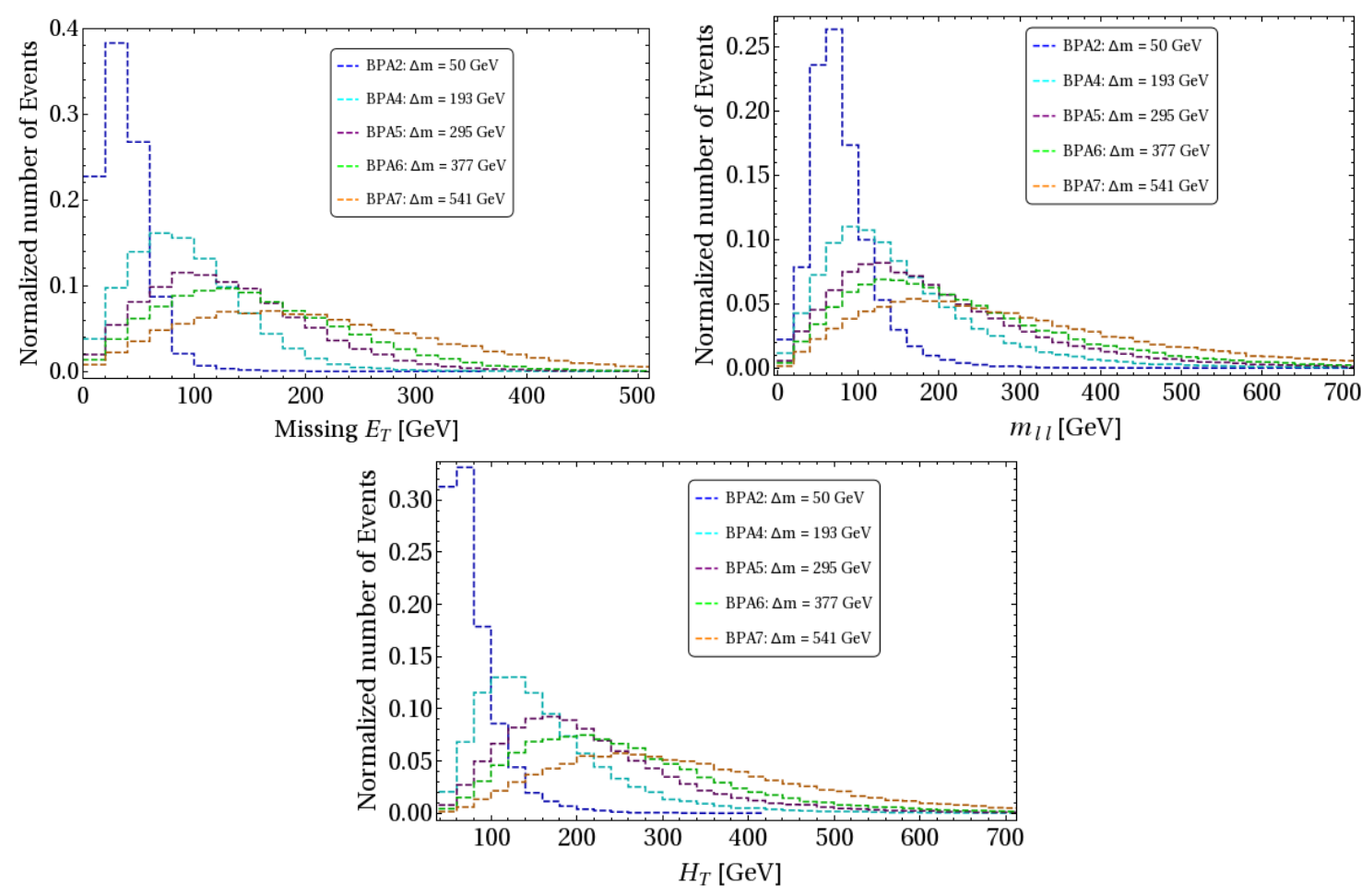

Figure 20. Missing energy $\left(\mathbb{E}_{T}\right)$, invariant mass of dilepton $\left(m_{\ell \ell}\right)$ and effective mass $\left(H_{T}\right)$ distributions of $\ell^{+} \ell^{-}+\left(\mathbb{E}_{T}\right)$ events from signal at LHC are shown at $\sqrt{s}=14 \mathrm{TeV}$. We have chosen different values of $\Delta m$ corresponding to different benchmark points as indicated in table 2 .

Therefore, for signal events with large $\Delta m$ can survive a large $\mathbb{E}_{T}$ cut while reducing the SM background significantly. This should be contrasted with low $\Delta m(\sim 50 \mathrm{GeV}, \mathrm{BPA} 2$ case), where the peak of missing energy falls within the same ballpark as those of SM backgrounds and therefore can not be separated. Therefore even if the signal cross-section is higher for such cases (as in the single component fermion DM case), the events are submerged into SM background. This feature is not very difficult to understand. With $\Delta m<m_{W}$, the $W$ decay is off-shell and $N^{ \pm}$momenta is shared amongst all the final state particles yielding a missing energy peak at lower value. For $\Delta m>m_{W}, W$ is produced on-shell and dominant momenta is carried by the dark matter $\left(N_{1}\right)$ as $m_{N_{1}}>m_{W}$. The higher the $\Delta m$ is, the higher is the available momenta for DM. This therefore yields missing energy peak at larger values with larger mass splitting $\Delta m$. We also note that such distinction is also possible with $H_{T}$ distribution. Again, the larger the $\Delta m$, the larger will be the available momenta for the leptons as well. Therefore, large $H_{T}$ cut can also reduce SM background retaining signals particularly for benchmark points with higher $\Delta m$. On the other hand, invariant mass cut can effectively reduce SM background events coming from $Z Z$ and $W Z$ background, when a cut is applied within the $Z$ mass window where the peak of the distribution lies. Therefore, to eliminate SM background from the signal event, we further employ some combination of the following cuts:

- $m_{\ell \ell}<\left|m_{z}-15\right|$ and $m_{\ell \ell}>\left|m_{z}+15\right|$,

- $H_{T}>100,200,300 \mathrm{GeV}$, 

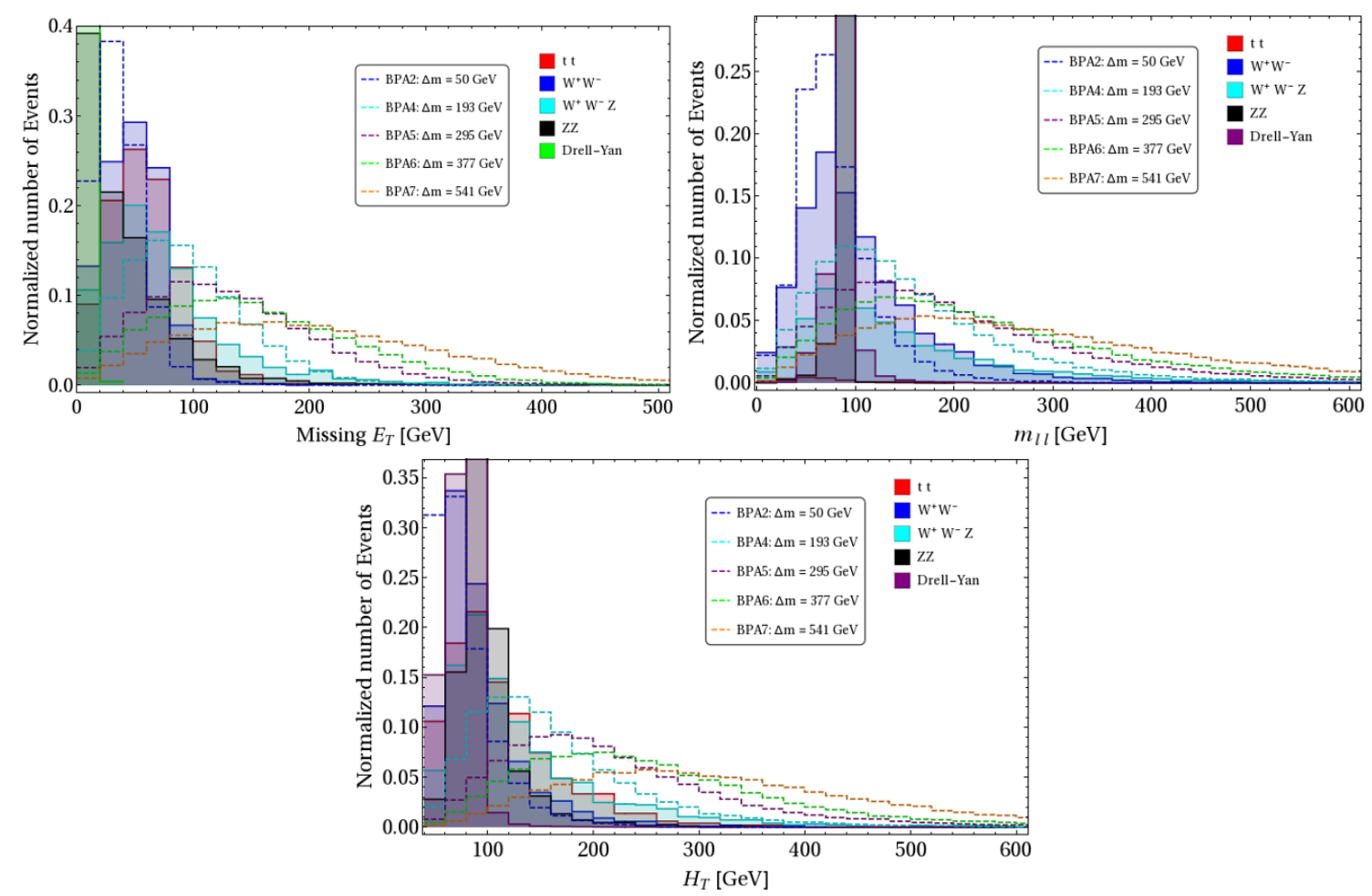

Figure 21. Missing energy $\left(\mathbb{E}_{T}\right)$, invariant mass of dilepton $\left(m_{\ell \ell}\right)$ and effective mass $\left(H_{T}\right)$ distributions of $\ell^{+} \ell^{-}+\left(\mathbb{E}_{T}\right)$ events from signal (Benchmark points as in table 2) and dominant SM background events at LHC with $\sqrt{s}=14 \mathrm{TeV}$.

- $\mathbb{E}_{T}>100,200,300 \mathrm{GeV}$.

Signal events with $\Delta m=\{50,101,193,295,377,541\}$ GeV corresponding to benchmark points BPA2, BPA4-BPA7 (as in table 2), are summarised in table 4, where the cut flow with different $H_{T}$ and $\mathbb{E}_{T}$ are furnished. The final state event rates $\left(N_{\text {eff }}\right)$ at a desired luminosity $\mathcal{L}$ is computed by:

$$
N_{\text {eff }}=\frac{\sigma_{p} n}{N} \times \mathcal{L},
$$

where $N$ is the simulated number of events and $n$ is the obtained final state events corresponding to production cross-section of $\sigma_{p}$. We see that although with larger $\Delta m$, the production cross-sections get diminished by the phase space suppression (as already pointed out in r.h.s. of figure 19), the shift in the peak of the distribution compensates it to ensure the survival of more number of signal events for such cases. With $\Delta m=101 \mathrm{GeV}$, the combination of $\mathbb{E}_{T}>200 \mathrm{GeV}$ and $H_{T}>100$, leaves with a very few events to be observed. $\mathcal{L}=100 \mathrm{fb}^{-1}$ turns out to be rather low to see the signals from such events and we need higher luminosity. The main take however is to note that only those cases where $\Delta m$ is large, has a prospect of discovery by reducing SM background through effective cuts, while those with small $\Delta m$ as in the single component framework is almost hopeless. The signal event rates can be contrasted with the SM background events with similar cut flow at $14 \mathrm{TeV}$ at LHC as detailed in table 5 . We also note that the limitation 


\begin{tabular}{|c|c|c|c|c|c|c|}
\hline $\mathrm{BPs}$ & $\Delta m(\mathrm{GeV})$ & $\sigma_{p p \rightarrow N^{+} N^{-}}(\mathrm{fb})$ & $\mathbb{E}_{T}(\mathrm{GeV})$ & $H_{T}(\mathrm{GeV})$ & $\sigma^{\mathrm{OSD}}(\mathrm{fb})$ & $N_{\mathrm{eff}}^{\mathrm{OSD}}$ \\
\hline \multirow{3}{*}{ BPA2 } & \multirow{3}{*}{50} & \multirow{3}{*}{1.73} & \multirow{3}{*}{$>100$} & $>100$ & 0.002 & $<1$ \\
\hline & & & & $>200$ & 0.001 & $<1$ \\
\hline & & & & $>300$ & 0.00 & 0 \\
\hline \multirow{6}{*}{ BPA3 } & \multirow{6}{*}{101} & \multirow{6}{*}{6.23} & \multirow[t]{3}{*}{$>100$} & $>100$ & 0.155 & 15 \\
\hline & & & & $>200$ & 0.045 & 4 \\
\hline & & & & $>300$ & 0.013 & 1 \\
\hline & & & \multirow[t]{3}{*}{$>200$} & $>100$ & 0.006 & 1 \\
\hline & & & & $>200$ & 0.005 & $<1$ \\
\hline & & & & $>300$ & 0.004 & $<1$ \\
\hline \multirow{6}{*}{ BPA4 } & \multirow{6}{*}{193} & \multirow{6}{*}{2.47} & \multirow[t]{3}{*}{$>100$} & $>100$ & 0.305 & 30 \\
\hline & & & & $>200$ & 0.138 & 14 \\
\hline & & & & $>300$ & 0.044 & 4 \\
\hline & & & \multirow[t]{3}{*}{$>200$} & $>100$ & 0.032 & 3 \\
\hline & & & & $>200$ & 0.031 & 3 \\
\hline & & & & $>300$ & 0.017 & 2 \\
\hline \multirow{9}{*}{ BPA5 } & \multirow{9}{*}{295} & \multirow{9}{*}{0.54} & \multirow[t]{3}{*}{$>100$} & $>100$ & 0.113 & 11 \\
\hline & & & & $>200$ & 0.075 & 7 \\
\hline & & & & $>300$ & 0.031 & 3 \\
\hline & & & \multirow[t]{3}{*}{$>200$} & $>100$ & 0.032 & 3 \\
\hline & & & & $>200$ & 0.031 & 3 \\
\hline & & & & $>300$ & 0.016 & 2 \\
\hline & & & \multirow[t]{3}{*}{$>300$} & $>100$ & 0.006 & 1 \\
\hline & & & & $>200$ & 0.006 & 1 \\
\hline & & & & $>300$ & 0.005 & $<1$ \\
\hline \multirow{9}{*}{ BPA6 } & \multirow{9}{*}{377} & \multirow{9}{*}{0.27} & \multirow[t]{3}{*}{$>100$} & $>100$ & 0.067 & 7 \\
\hline & & & & $>200$ & 0.052 & 5 \\
\hline & & & & $>300$ & 0.027 & 3 \\
\hline & & & \multirow[t]{3}{*}{$>200$} & $>100$ & 0.027 & 3 \\
\hline & & & & $>200$ & 0.027 & 3 \\
\hline & & & & $>300$ & 0.016 & 2 \\
\hline & & & \multirow[t]{3}{*}{$>300$} & $>100$ & 0.007 & 1 \\
\hline & & & & $>200$ & 0.007 & 1 \\
\hline & & & & $>300$ & 0.006 & 1 \\
\hline & & & $>100$ & $>100$ & 0.017 & 2 \\
\hline & & & & $>200$ & 0.015 & 1 \\
\hline & & & & $>300$ & 0.011 & 1 \\
\hline BPA7 & 541 & 0.06 & $>200$ & $>100$ & 0.011 & 1 \\
\hline & & & & $>200$ & 0.010 & 1 \\
\hline & & & & $>300$ & 0.008 & 1 \\
\hline
\end{tabular}

Table 4. Signal events for few selected benchmark points (BPA2-BPA7, see table 2) with $\sqrt{s}=$ $14 \mathrm{TeV}$ at the LHC for the luminosity $\mathcal{L}=100 \mathrm{fb}^{-1}$ after $\mathbb{E}_{T}, H_{T}$ and $m_{\ell \ell}$ cuts. 


\begin{tabular}{|c|c|c|c|c|c|}
\hline SM Backgrounds & $\sigma_{p p \rightarrow S M}(\mathrm{fb})$ & $\mathbb{E}_{T}(\mathrm{GeV})$ & $H_{T}(\mathrm{GeV})$ & $\sigma^{\mathrm{OSD}}(\mathrm{fb})$ & $N_{\text {eff }}^{\mathrm{OSD}}$ \\
\hline \multirow{9}{*}{$t \bar{t}$} & \multirow{9}{*}{$814.78 \times 10^{3}$} & \multirow[t]{3}{*}{$>100$} & $>100$ & 17.11 & 1711 \\
\hline & & & $>200$ & 2.44 & 244 \\
\hline & & & $>300$ & $<0.81$ & $<1$ \\
\hline & & \multirow[t]{3}{*}{$>200$} & $>100$ & $<0.81$ & $<1$ \\
\hline & & & $>200$ & $<0.81$ & $<1$ \\
\hline & & & $>300$ & $<0.81$ & $<1$ \\
\hline & & \multirow[t]{3}{*}{$>300$} & $>100$ & $<0.81$ & $<1$ \\
\hline & & & $>200$ & $<0.81$ & $<1$ \\
\hline & & & $>300$ & $<0.81$ & $<1$ \\
\hline \multirow{9}{*}{$W^{+} W^{-}$} & \multirow{9}{*}{$100.06 \times 10^{3}$} & \multirow[t]{3}{*}{$>100$} & $>100$ & 20.51 & 2051 \\
\hline & & & $>200$ & 10.01 & 1001 \\
\hline & & & $>300$ & 2.00 & 200 \\
\hline & & \multirow[t]{3}{*}{$>200$} & $>100$ & 2.00 & 200 \\
\hline & & & $>200$ & 2.00 & 200 \\
\hline & & & $>300$ & 0.50 & 50 \\
\hline & & \multirow[t]{3}{*}{$>300$} & $>100$ & $<0.50$ & $<1$ \\
\hline & & & $>200$ & $<0.50$ & $<1$ \\
\hline & & & $>300$ & $<0.50$ & $<1$ \\
\hline \multirow{9}{*}{$Z Z$} & \multirow{9}{*}{$14.03 \times 10^{3}$} & \multirow[t]{3}{*}{$>100$} & $>100$ & 0.21 & 21 \\
\hline & & & $>200$ & 0.14 & 14 \\
\hline & & & $>300$ & 0.07 & 7 \\
\hline & & \multirow[t]{3}{*}{$>200$} & $>100$ & $<0.07$ & $<1$ \\
\hline & & & $>200$ & $<0.07$ & $<1$ \\
\hline & & & $>300$ & $<0.07$ & $<1$ \\
\hline & & \multirow[t]{3}{*}{$>300$} & $>100$ & $<0.07$ & $<1$ \\
\hline & & & $>200$ & $<0.07$ & $<1$ \\
\hline & & & $>300$ & $<0.07$ & $<1$ \\
\hline \multirow{9}{*}{$W^{+} W^{-} Z$} & \multirow{9}{*}{$0.16 \times 10^{3}$} & \multirow[t]{3}{*}{$>100$} & $>100$ & 0.17 & 17 \\
\hline & & & $>200$ & 0.09 & 9 \\
\hline & & & $>300$ & 0.03 & 3 \\
\hline & & \multirow[t]{3}{*}{$>200$} & $>100$ & 0.04 & 4 \\
\hline & & & $>200$ & 0.04 & 4 \\
\hline & & & $>300$ & 0.02 & 2 \\
\hline & & \multirow[t]{3}{*}{$>300$} & $>100$ & 0.01 & 1 \\
\hline & & & $>200$ & 0.01 & 1 \\
\hline & & & $>300$ & 0.01 & 1 \\
\hline
\end{tabular}

Table 5. Dominant SM background contribution to $\ell^{+} \ell^{-}+\left(\mathbb{E}_{T}\right)$ signal events with $\sqrt{s}=14 \mathrm{TeV}$ at the LHC for luminosity $\mathcal{L}=100 \mathrm{fb}^{-1}$ after $\mathbb{E}_{T}, H_{T}$ and $m_{\ell \ell}$ cuts. The variation of effective number of final state background events with cut-flow are also tabulated. 

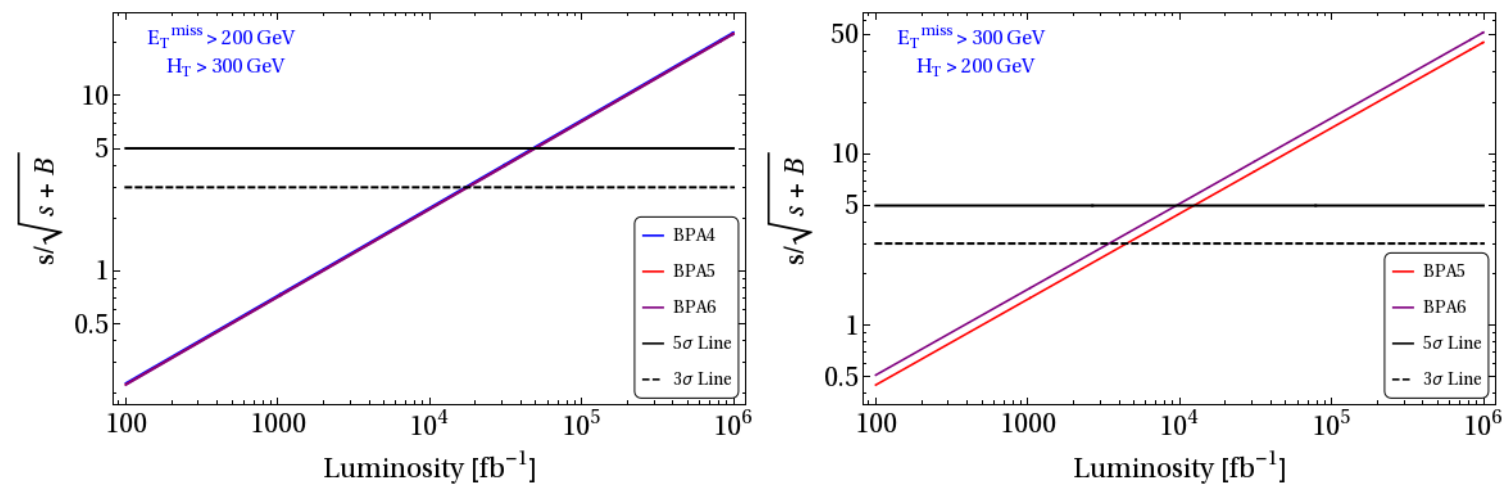

Figure 22. Signal significance $\sigma=\frac{S}{\sqrt{S+B}}$ of OSD events for select few benchmark points (see table 2) at LHC with $E_{C M}=14 \mathrm{TeV}$ as a function of integrated luminosity. Different combinations of $E_{T}^{\prime}, H_{T}$ cuts are chosen in left and right panel (mentioned in inset). $3 \sigma$ (black dashed) and $5 \sigma$ (black thick) lines are indicated as references.

in warranting any final state event with number of simulated points yield a limit on the effective background cross-section as indicated in the table. We see that the dominant SM backgrounds can be tamed down significantly with a combination of $\mathbb{E}_{T}$ and $H_{T}$ cut. The reach of the signal significance $\sigma=\frac{S}{\sqrt{S+B}}$ is plotted with integrated luminosity $\mathcal{L}$ for selected benchmark points with two different combinations of $\mathbb{E}_{T}$ and $H_{T}$ cut in left and right panel of figure 22 . It shows that $5 \sigma$ significance can be reached with luminosity as high as $\sim 10^{4} \mathrm{fb}^{-1}$.

We also note here that small $\Delta m$ along with small $\sin \theta$ predicts a delay in the decay of the charged fermion, yielding displaced vertex or stable charge track signature and serves as a characteristic signal for the fermion dark sector with singlet-doublet mixing, as has already been noted in ref. [35]. However, in that case, signal excess in dilepton channel can not be seen. On the contrary, with large $\Delta m$, when excess in opposite sign dilepton events can be seen, the decay of the charged fermion is quick and therefore no displaced vertex signature can be observed. Therefore the signal of singlet-doublet fermion DM in presence of a second lighter DM component has a complementarity to that of the same DM in a single component framework as far as collider search is concerned. The presence of a heavy scalar (as illustrated in section 5) doesn't of course change the fermion DM signal discussed here, but allows one to choose the scalar DM in a large mass range.

\section{Possible implications to inflation and reheating}

In this section, we comment briefly on the possibility of production of dark sector particles in the early Universe. It is usually assumed that the early Universe has gone through a period inflation driven by a scalar field, the so called inflaton. Subsequently the inflaton decays perturbatively (nonperturbatively) to bring back a thermal bath, the so called reheating (preheating) phase, with a temperature $T_{R}>4 \mathrm{MeV}$ to pave a path for BigBang nucleosynthesis (BBN). See for a review [53, 54]. During the reheating (preheating) phase all the elementary particles, including dark sector, are assumed to be produced. 
The process of reheating is quite model dependent and accordingly the temperature $T_{R}$ of thermal bath is set in a large range.

It has been pointed out that during Inflation, the SM Higgs boson may develop a non-zero and large vev $h_{I} \sim H_{I}$ [55], where $H_{I}$ is the Hubble scale at the end of inflation. Therefore, the particles which couple to the Higgs will also acquire very high mass during this period. This may result in a kinematic blocking of the inflaton decay if the mass of the inflaton is lighter that than the decay products induced by the non-zero Higgs vev [55]. The phenomena is involved and model dependent. We just provide a brief sketch of the main idea. The presence of the scalar DM which couples to Higgs in our scenario through Higgs portal, may add to the phenomena. A simple illustration of the above situation can be made by looking into the perturbative inflaton decay neglecting backreaction. If we assume a simple inflaton $(\phi)$ potential given by $V_{\phi}=m_{\phi}^{2} \phi^{2} / 2$, the perturbative reheating temperature $\left(T_{R}\right)$ is obtained through the solution of the following coupled Boltzmann equations:

$$
\begin{aligned}
\dot{\rho_{\phi}}+3 H \rho_{\phi} & =-\Gamma_{\phi} \rho_{\phi}, \\
\dot{\rho_{R}}+3 H \rho_{R} & =\Gamma_{\phi} \rho_{\phi},
\end{aligned}
$$

where $\rho_{\phi}$ is the density of the inflaton and $\rho_{R}$ is the density of radiation resulting from the decay of the inflaton with decay width $\Gamma_{\phi} . H$ is the Hubble constant with $H^{2}=\frac{8 \pi}{3}\left(\rho_{\phi}+\rho_{R}\right)$. In presence of the DM, the inflaton also decays to DM in addition to SM particles and the total decay width is given by:

$$
\Gamma_{\phi}=\Gamma_{0}\left(1-\frac{4 m_{f}^{2}}{m_{\phi}^{2}}\right)^{3 / 2} \Theta\left(m_{\phi}^{2}-4 m_{f}^{2}\right)+\Gamma_{0}\left(1-\frac{4 m_{s}^{2}}{m_{\phi}^{2}}\right)^{3 / 2} \Theta\left(m_{\phi}^{2}-4 m_{s}^{2}\right) .
$$

In above equation, for simplicity, we just incorporate the decay to SM fermions $(f)$ and to the scalar DM $S . \Gamma_{0}$ denotes the decay width at zero mass limit. The mass term for the SM fermion and DM are generated from Yukawa interactions ( $y$ and $\lambda_{S H}$ ) followed by the large vev $\left(h_{I}\right)$ that Higgs acquires during inflation and will be given by:

$$
m_{f}^{2}=\frac{1}{2} y^{2} h_{I}^{2} ; m_{S}^{2}=\frac{1}{2} \lambda_{S H}^{2} h_{I}^{2}
$$

The $\Theta$ function in eq. (7.2) denotes the phase space blocking. Depending on whichever is lighter between $m_{f}$ and $m_{S}$, the effective blocking condition for the inflaton decay (assuming $m_{S}<m_{f}$ ) reads:

$$
\frac{h_{I}^{2}}{m_{\phi}^{2}}>\frac{1}{2 \lambda_{S H}^{2}} .
$$

As a result the reheating temperature $T_{R}$ can drop significantly and can be even be less than the Higgs mass depending on the coupling and vev. The delay in reheating may alter the CMB spectrum in terms of the spectral index of density perturbation $\left(n_{s}\right)$ and tensorto-scalar ratio $(r)$ or affect the heavy particle production. However, one should note here that the maximum temperature $T_{\max }$ during reheating can be much larger than reheating 
temperature $T_{R}$. During reheating, the temperature rises to $T_{\max }$ and then falls to $T_{R}$, see for example, [56]:

$$
T_{\max }=0.6 g_{*}^{-1 / 4}\left(\Gamma_{\phi} M_{\mathrm{Pl}}\right)^{1 / 4} M_{I}^{1 / 2},
$$

where $M_{I}=V_{I}^{1 / 4}, V_{I}$ depicts the energy density at $t_{o s c}$. Depending on the model, maximum temperature can be as high as $T_{\max } \sim 10^{3} T_{R}$. As a result, the heavy particles (including dark sector particles $\chi_{1}, \chi_{2}, N, S$ etc.) in general can be produced during reheating phase itself. Once these particles are produced, irrespective of their initial number density, they can easily thermalise due to their coupling with the SM Higgs and other SM particles. For instance in our case $N$ is a doublet. So it can be easily thermalise due to its gauge coupling. On the other hand, $\chi_{1}$, and $S$ are singlet under the SM gauge group. However, these particles couple to the SM Higgs through (large) Yukawa interaction ( $Y_{1}$ and $\lambda_{S H}$ respectively). Therefore, the dark sector particles in our case are no more in danger being over produced even if the kinematic blocking effects in a lower reheat temperature as discussed above.

\section{Summary}

The dark sector of the universe is still a mystery to us. In this work, we have discussed a possible two component (WIMP-like) DM scenario with a vector like fermion (an admixture of a singlet and a doublet) and a scalar singlet stabilised by $\mathcal{Z}_{2} \times \mathcal{Z}_{2}^{\prime}$ symmetry. The proposed scenario crucially addresses the possibility of DM-DM interaction between fermion and scalar DM candidates through another heavy vectorlike fermion singlet which acts as mediator. We show that in absence of the mediator (which means the absence of $t$ - channel heavy fermion mediated DM-DM interaction), both fermion and scalar DM components behave like two decoupled single component DMs. This is due to suppressed s-channel Higgs mediated interaction between the DM components. In such a situation, both of the sector turns out to fill up the corresponding under-abundant regions to add to the observed relic density. Although such a non interacting situation satisfy observed DM relic density, the direct search limit (XENON1T) rules-out most of the parameter space, particularly for the scalar DM to a very heavy mass $\gtrsim 1 \mathrm{TeV}$. For fermion DM, the necessity of coannihilation contribution limits the mass difference with the charged doublet component to a small value $(\lesssim 12 \mathrm{GeV})$.

However, in presence of a heavy fermion mediated $t$-channel DM-DM conversion, with moderate values of mediator mass $\sim 500 \mathrm{GeV}$, the freeze out and relic density of DM components get affected significantly. The change is observed mostly in the relic density of the heavier DM component, which has the liberty of annihilating to the lighter DM, unconstrained by direct search limit; while lighter DM component behaves mostly as in single component framework. So, by allowing DM-DM conversion in the interacting picture, we open up large parameter space allowed by both relic density and direct search bounds which otherwise yields over-abundance in non-interacting cases. For fermion DM (when it is heavier than scalar DM), large $\Delta m$ regions become allowed, but scalar DM is restricted to the Higgs resonance region. In presence of a heavy scalar, which helps co-annihilating 

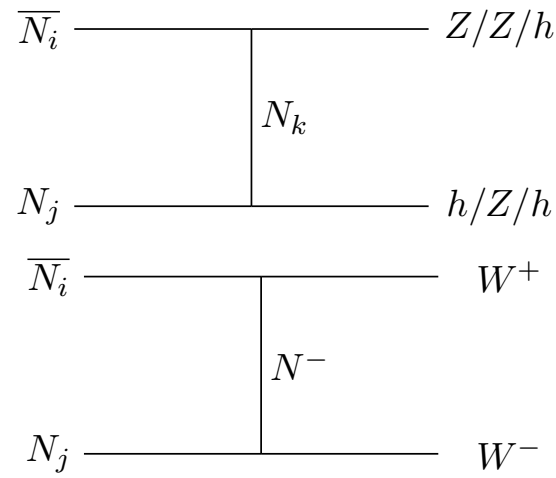
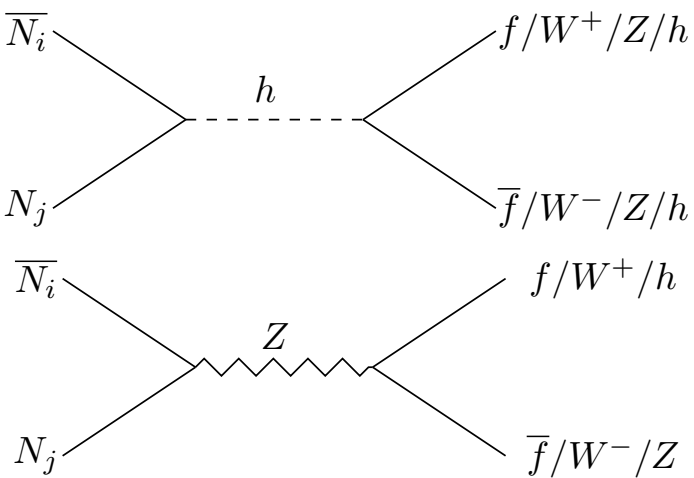

Figure 23. Annihilation $(i=j)$ and Co-annihilation $(i \neq j)$ of fermion DM. Here $(i, j, k=1,2)$.

the scalar DM component, allow a larger mass range for scalar DM even when it is lighter than fermion DM. On the other hand, when scalar DM is heavier than fermion DM, DMDM conversion allows the presence of smaller Higgs portal couplings, hiding the scalar DM from direct search to allow a larger mass range upto $\mathrm{TeV}$ and beyond.

The work also demonstrates the importance of DM-DM conversion in seeing signals of a dark sector at LHC in relic density and direct search allowed parameter space. In the model, fermion dark sector is composed of a doublet and a singlet. Hence, the charged companions can be produced at LHC which yields hadronically quiet oppsite sign dilepton events plus missing energy through their decays to fermion DM. However, in a single component framework, relic density and direct search constraints restrict the fermion DM to have a small mass difference with the charged companion $(\Delta m)$, which makes the signal submerged into SM background. On the contrary, in presence of a lighter DM component and an effective DM-DM conversion, $\Delta m$ can be large, which can segregate the signal from SM background by a combination of large missing energy and effective mass cuts as detailed in the analysis. The discovery limit of such a signal still might be delayed to an integrated luminosity $\sim 10^{4} \mathrm{fb}^{-1}$.

\section{Acknowledgments}

The authors would like to acknowledge discussions with Dr. Nirakar Sahoo, Dr. Debaprasad Maity and Dr. Pankaj Saha. PG would like to thank Dr. Rashidul Islam and Basabendu Barman for fruitful discussions and MHRD, Government of India for research fellowship. S.B. would like to acknowledge the DST-INSPIRE research grant IFA13-PH-57 at IIT Guwahati.

\section{A Single component vector-like fermion DM}

The freeze-out of $N_{1} \mathrm{DM}$ is controlled by the annihilation and co-annihilation channels as shown in figures $23,24,25$. This is mainly driven by gauge mediation and Higgs mediation apart from the $t$-channel heavy fermion $\left(N_{2}, N^{ \pm}\right)$mediation. 


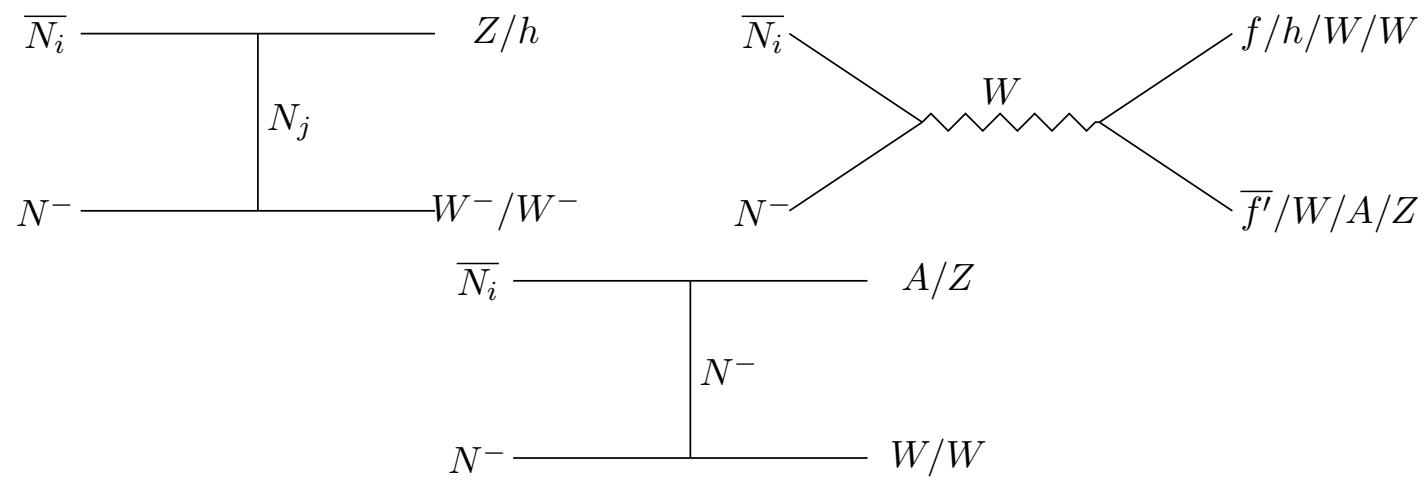

Figure 24. Co-annihilation process of $N_{i}(i=1,2)$ with the charge component $N^{-}$to SM particles.
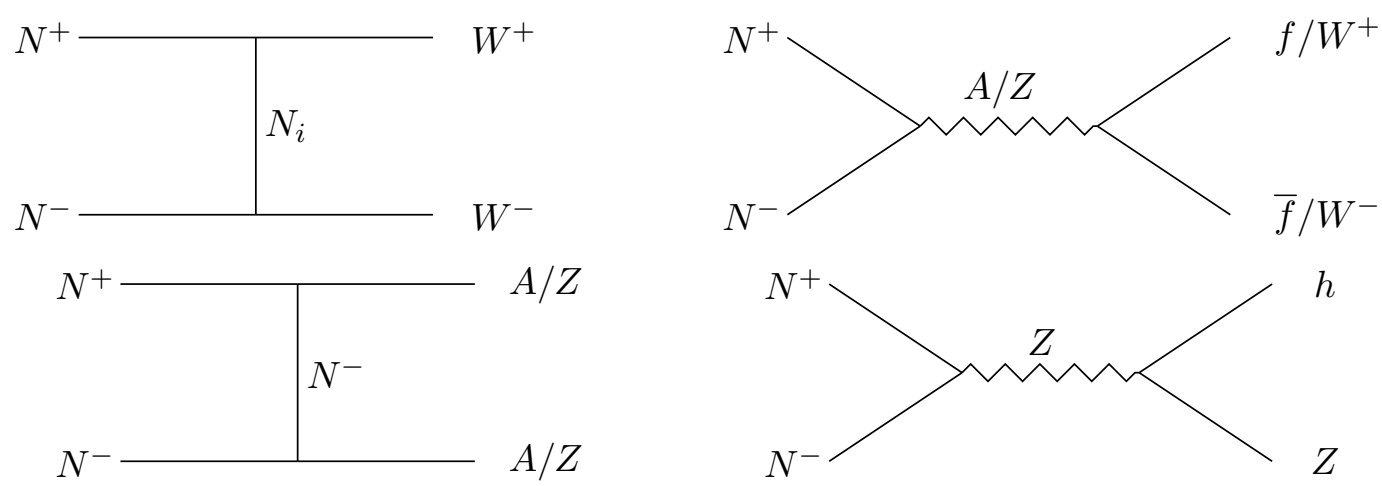

Figure 25. Co-Annihilation process of charged fermions $N^{ \pm}$to SM particles in final states.

Relic density of vector like fermion DM is then governed by the effective number changing cross-section following [57],

$$
\begin{aligned}
& \langle\sigma v\rangle_{N_{1}}^{\mathrm{eff}}=\frac{g_{1}^{2}}{g_{\mathrm{eff}}^{2}}\langle\sigma v\rangle_{\overline{N_{1}} N_{1}}+\frac{2 g_{1} g_{2}}{g_{\mathrm{eff}}^{2}}\langle\sigma v\rangle_{\overline{N_{1}} N_{2}}\left(1+\frac{\Delta m}{m_{N_{1}}}\right)^{\frac{3}{2}} e^{-x \frac{\Delta m}{m_{N_{1}}}} \\
& +\frac{2 g_{1} g_{3}}{g_{\text {eff }}^{2}}\langle\sigma v\rangle_{\overline{N_{1}} N^{-}}\left(1+\frac{\Delta m}{m_{N_{1}}}\right)^{\frac{3}{2}} e^{-x \frac{\Delta m}{m_{N_{1}}}} \\
& +\frac{2 g_{2} g_{3}}{g_{\text {eff }}^{2}}\langle\sigma v\rangle_{N^{+} N_{2}}\left(1+\frac{\Delta m}{m_{N_{1}}}\right)^{3} e^{-2 x \frac{\Delta m}{m_{N_{1}}}} \\
& +\frac{g_{2}^{2}}{g_{\mathrm{eff}}^{2}}\langle\sigma v\rangle_{N_{2} N_{2}}\left(1+\frac{\Delta m}{m_{N_{1}}}\right)^{3} e^{-2 x \frac{\Delta m}{m_{N_{1}}}} \\
& +\frac{g_{3}^{2}}{g_{\mathrm{eff}}^{2}}\langle\sigma v\rangle_{N^{+} N^{-}}\left(1+\frac{\Delta m}{m_{N_{1}}}\right)^{3} e^{-2 x \frac{\Delta m}{m_{N_{1}}}}
\end{aligned}
$$

In above equation, $g_{\text {eff }}$, defined as effective degrees of freedom, is given by

$$
g_{\text {eff }}=g_{1}+g_{2}\left(1+\frac{\Delta m}{m_{N_{1}}}\right)^{\frac{3}{2}} e^{-x \frac{\Delta m}{m_{N_{1}}}}+g_{3}\left(1+\frac{\Delta m}{m_{N_{1}}}\right)^{\frac{3}{2}} e^{-x \frac{\Delta m}{m_{N_{1}}}},
$$



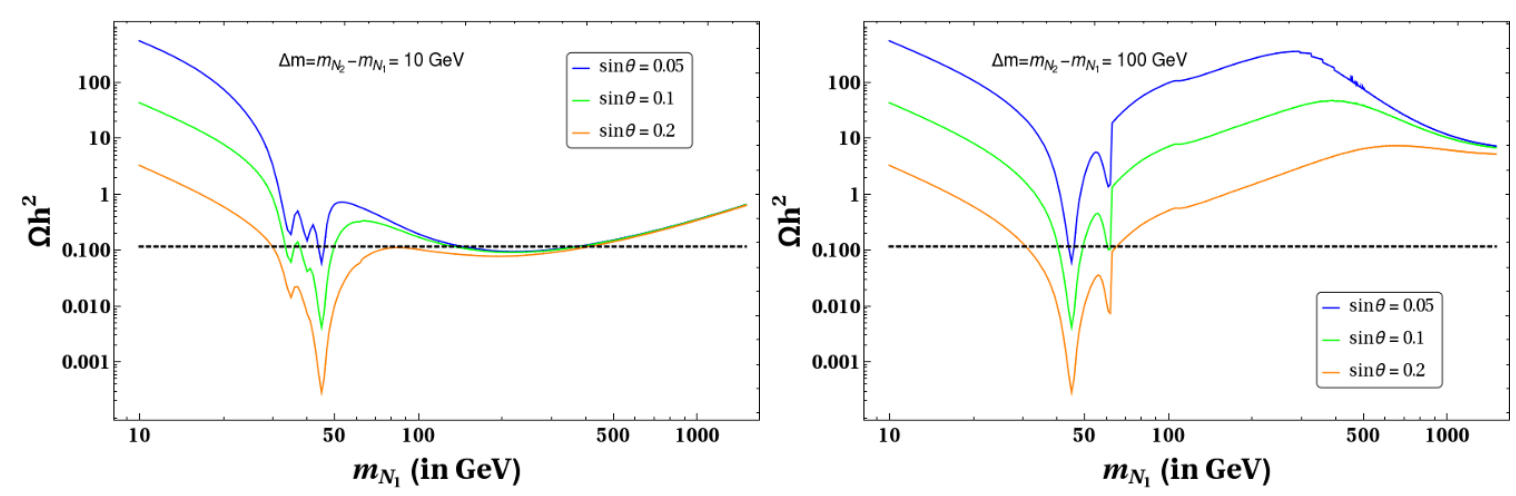

Figure 26. Relic density of $N_{1}$ as a function of DM mass, $m_{N_{1}}$ with different mixing angle, $\sin \theta=0.05$ (blue), $\sin \theta=0.1$ (green) and $\sin \theta=0.2$ (orange). Each plot corresponds to fixed $\Delta m:=10 \mathrm{GeV}$ (left), $100 \mathrm{GeV}$ (right). Black dashed line indicates observed relic density $0.1133 \leq$ $\Omega_{D M} h^{2} \leq 0.1189$.

where $g_{1}, g_{2}$ and $g_{3}$ are the degrees of freedom of $N_{1}, N_{2}$ and $\mathrm{N}^{-}$respectively and $x=$ $x_{f}=\frac{m_{N_{1}}}{T_{f}}$, where $T_{f}$ is the freeze out temperature of $N_{1}$. Then relic density will be given by $[7,20]$ :

$$
\Omega h^{2}=\frac{854.45 \times 10^{-13}}{\sqrt{106.7}} \frac{x_{f}}{\langle\sigma v\rangle_{N_{1}}^{\text {eff }}},
$$

assuming $x_{f} \sim 20$.

Variation of relic density of fermion DM is shown as a function of DM mass, for a fixed $\Delta m=10 \mathrm{GeV}$ (left panel of the figure 26) and $100 \mathrm{GeV}$ (right panel of the figure 26) and different choices of mixing angle, $\sin \theta$. We note that the annihilation cross-section is larger when we take larger values of $\sin \theta$, due to larger $\mathrm{SU}(2)$ component, resulting smaller relic density. The resonance drop at $m_{Z} / 2$ and at $m_{h} / 2$ is observed due to $s$-channel $Z$ and $H$ mediated contributions. For $\Delta m=100 \mathrm{GeV}$, due to smaller co-annihilation contribution relic density increases compared to $\Delta m=10 \mathrm{GeV}$ case.

\section{B Higgs invisible decay constraint}

When masses of DMs are smaller than the Higgs mass i.e. $m_{D M}<m_{h} / 2$, then Higgs can decay to DM (invisible particles) and will contribute to invisible decay width. LHC data puts strong constraint on the invisible branching fraction of Higgs as $\operatorname{Br}(h \rightarrow i n v)<$ 0.24 [42]. This can be interpreted as follows:

$$
\begin{aligned}
\operatorname{Br}(h \rightarrow i n v .) & <0.24 \\
\frac{\Gamma(h \rightarrow i n v .)}{\Gamma(h \rightarrow S M)+\Gamma(h \rightarrow i n v .)} & <0.24
\end{aligned}
$$

where $\Gamma(h \rightarrow S M)=4.2 \mathrm{MeV}$ for SM Higgs (with mass $m_{h}=125.09 \mathrm{GeV}$ ) is measured at LHC [42]. This then yields,

$$
\Gamma(h \rightarrow \text { inv. })<1.32 \mathrm{MeV} .
$$



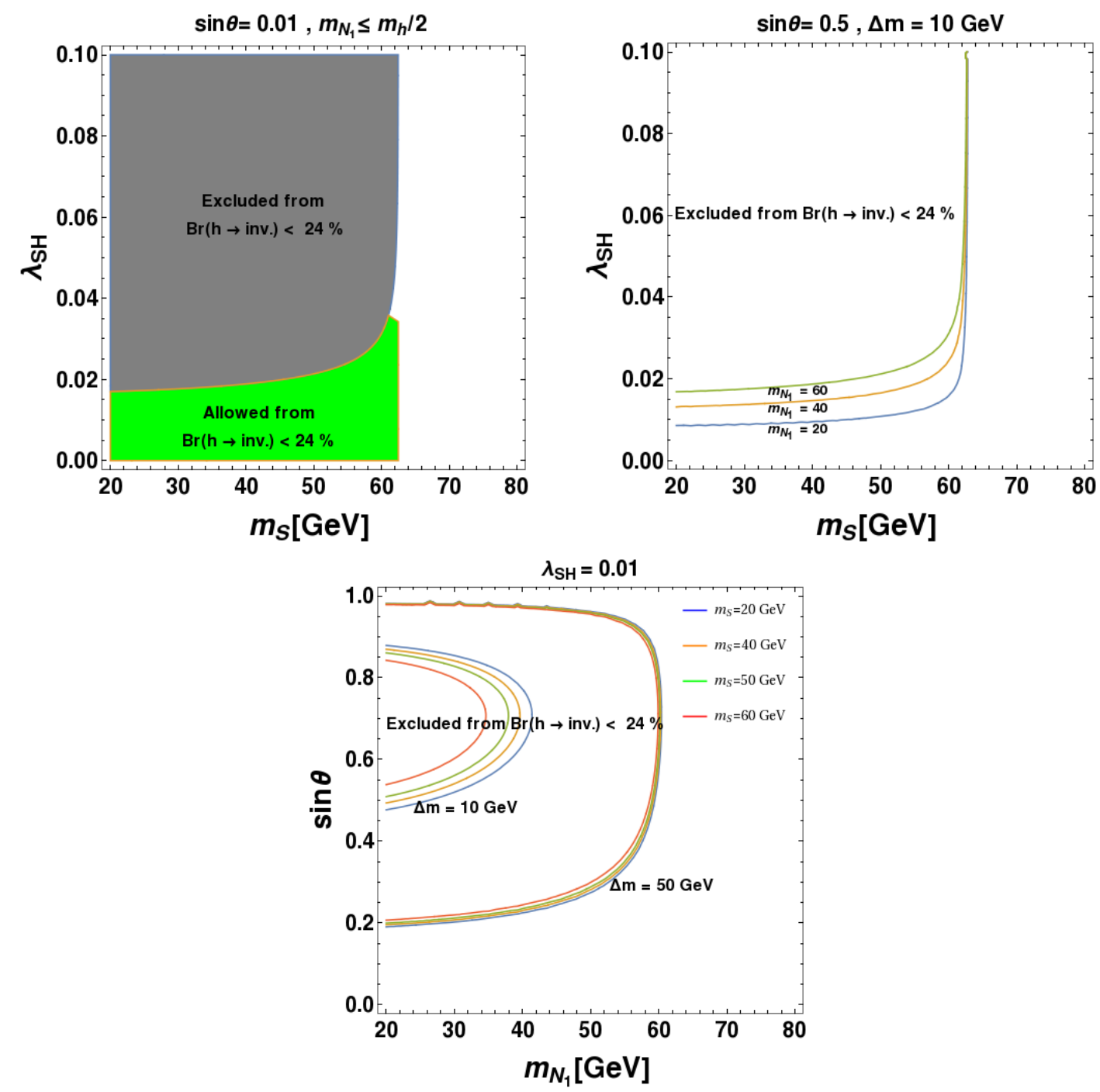

Figure 27. Constraints on scalar and fermion DM from Higgs invisible branching ratio $\mathrm{Br}(h \rightarrow$ $i n v.)<0.24$ [42] in $m_{S}-\lambda_{S H}$ plane (top panel) and $m_{N_{1}}-\sin \theta$ plane (bottom panel) keeping other parameter fixed (mentioned in the figure inset).

In our two component DM scenario, the invisible decay may have two contributions if both $m_{N_{1}}, m_{S}<m_{h} / 2$ :

$$
\Gamma(h \rightarrow i n v .)=\Gamma\left(h \rightarrow \overline{N_{1}} N_{1}\right)+\Gamma(h \rightarrow S S) .
$$

The decay width of Higgs to $S$ and $\mathrm{N}_{1}$ can easily be calculated as:

$$
\begin{aligned}
\Gamma_{h \rightarrow S S} & =\frac{\lambda_{S H}^{2} v^{2}}{32 \pi m_{h}^{2}} \sqrt{m_{h}^{2}-4 m_{S}^{2}} \Theta\left(m_{h}-2 m_{S}\right), \\
\Gamma_{h \rightarrow \overline{N_{1}} N_{1}} & =\frac{1}{16 \pi}\left(Y_{1} \sin 2 \theta\right)^{2} m_{h}\left(1-\frac{4 m_{N_{1}}^{2}}{m_{h}^{2}}\right)^{\frac{3}{2}} \Theta\left(m_{h}-2 m_{N_{1}}\right) .
\end{aligned}
$$

Invisible Higgs decay constraint from eq. (B.2) together with eq. (B.3) and eq. (B.4) is shown in figure 27 . In top left panel of figure 27 , the constraint is shown in $m_{S}-\lambda_{S H}$ plane. 
Here, the green region is allowed from Higgs invisible decay while grey region excluded for a fixed $\sin \theta=0.01$. The allowed (or excluded) region remains almost unchanged for any fermion DM mass $\left(m_{N_{1}}<m_{h} / 2\right)$ and $\Delta m$ for the small $\sin \theta$ due to negligible contribution of $\Gamma\left(h \rightarrow \overline{N_{1}} N_{1}\right)$. In the top right panel we consider larger mixing angle, $\sin \theta=0.5$. As the contribution the contribution of $\Gamma\left(h \rightarrow \overline{N_{1}} N_{1}\right)$ plays a important role to $\Gamma(h \rightarrow i n v$. $)$. And therefore, choices of other parameters like $m_{N_{1}}, \Delta m$ becomes relevant. The inner region of each contour in $m_{S}-\lambda_{S H}$ plane (top right panel) is excluded from Higgs invisible decay constraint [42]. Note here however that such large $\sin \theta(=0.5)$ is disfavoured from direct search bounds $[9,10]$. In the bottom panel we have shown excluded region in $m_{N_{1}}-\sin \theta$ plane keeping other parameters, $m_{S}, \lambda_{S H}$ and $\Delta m$ fixed. Similarly here the inner region of each contour line (which corresponds to different fixed values of $\Delta m$ and scalar DM mass $\left(m_{S}\right)$, depicted in the figure) is excluded from Higgs invisible decay [42].

\section{Invisible decay constraint of $Z$}

As the fermion DM has a doublet component in it, it has $Z$ mediated interaction. Hence, if fermion DM mass is below $m_{Z} / 2$, then $Z$ can invisibly decay to dark particles. From current observation, invisible decay width of $Z$ is strongly constrained. The upper limit of invisible $\mathrm{Z}$ decay width is following [42]:

$$
\Gamma(Z \rightarrow \text { inv. }) \leq 499.0 \pm 1.5 \mathrm{MeV},
$$

where in our model,

$$
\begin{aligned}
\Gamma(Z \rightarrow i n v .) & =\Gamma\left(Z \rightarrow \overline{N_{1}} N_{1}\right) \\
& =\frac{1}{48 \pi}\left(\frac{g \sin ^{2} \theta}{\cos \theta_{W}}\right)^{2} m_{Z}\left(1+\frac{2 m_{N_{1}}^{2}}{m_{Z}^{2}}\right) \sqrt{1-\frac{4 m_{N_{1}}^{2}}{m_{Z}^{2}}} \Theta\left(m_{Z}-2 m_{N_{1}}\right)
\end{aligned}
$$

Invisible decay of $Z$ mainly depend on mixing angle $\sin \theta$. Choice of small mixing angle, with $\sin \theta<0.1$ is preferable from direct search bound in which all fermion DM mass $m_{N_{1}}<m_{Z} / 2$ is allowed from invisible decay width of $Z$ [20]. We note that as the scalar DM component is a gauge singlet, it doesn't have a $Z$ mediated interaction and therefore no constraint from invisible $Z$ decay applies to it.

Open Access. This article is distributed under the terms of the Creative Commons Attribution License (CC-BY 4.0), which permits any use, distribution and reproduction in any medium, provided the original author(s) and source are credited.

\section{References}

[1] V.C. Rubin, Optical observations of radio galaxies and quasi-stellar objectsOptical observations of radio galaxies and quasi-stellar radiosources, in Hautes Energies en Astrophysique: Proceedings, Ecole d'Eté de Physique Théorique, Les Houches, France, 1966, vol. 1, (1967) pp. 133-152.

[2] V.C. Rubin and W.K. Ford Jr., Rotation of the Andromeda Nebula from a Spectroscopic Survey of Emission Regions, Astrophys. J. 159 (1970) 379 [InSPIRE]. 
[3] W. Hu and S. Dodelson, Cosmic microwave background anisotropies, Ann. Rev. Astron. Astrophys. 40 (2002) 171 [astro-ph/0110414] [INSPIRE].

[4] G. Bertone, D. Hooper and J. Silk, Particle dark matter: Evidence, candidates and constraints, Phys. Rept. 405 (2005) 279 [hep-ph/0404175] [INSPIRE].

[5] WMAP collaboration, Nine-Year Wilkinson Microwave Anisotropy Probe (WMAP) Observations: Cosmological Parameter Results, Astrophys. J. Suppl. 208 (2013) 19 [arXiv: 1212.5226] [INSPIRE].

[6] Planck collaboration, Planck 2013 results. XVI. Cosmological parameters, Astron. Astrophys. 571 (2014) A16 [arXiv:1303.5076] [INSPIRE].

[7] E.W. Kolb and M.S. Turner, The Early Universe, Front. Phys. 69 (1990) 1 [INSPIRE].

[8] J. Billard, L. Strigari and E. Figueroa-Feliciano, Implication of neutrino backgrounds on the reach of next generation dark matter direct detection experiments, Phys. Rev. D 89 (2014) 023524 [arXiv: 1307.5458 ] [INSPIRE].

[9] LUX collaboration, Limits on spin-dependent WIMP-nucleon cross section obtained from the complete LUX exposure, Phys. Rev. Lett. 118 (2017) 251302 [arXiv:1705. 03380] [INSPIRE].

[10] XENON collaboration, Dark Matter Search Results from a One Ton-Year Exposure of XENON1T, Phys. Rev. Lett. 121 (2018) 111302 [arXiv:1805.12562] [INSPIRE].

[11] XENON collaboration, Physics reach of the XENON1T dark matter experiment, JCAP 04 (2016) 027 [arXiv:1512.07501] [INSPIRE].

[12] PandaX-II collaboration, Dark Matter Results From 54-Ton-Day Exposure of PandaX-II Experiment, Phys. Rev. Lett. 119 (2017) 181302 [arXiv:1708.06917] [INSPIRE].

[13] Q.-H. Cao, E. Ma, J. Wudka and C.P. Yuan, Multipartite dark matter, arXiv:0711.3881 [INSPIRE].

[14] S. Bhattacharya, A. Drozd, B. Grzadkowski and J. Wudka, Two-Component Dark Matter, JHEP 10 (2013) 158 [arXiv: 1309.2986] [INSPIRE].

[15] A. Biswas, D. Majumdar, A. Sil and P. Bhattacharjee, Two Component Dark Matter: A Possible Explanation of $130 \mathrm{GeV} \gamma-$ Ray Line from the Galactic Centre, JCAP 12 (2013) 049 [arXiv: 1301.3668] [INSPIRE].

[16] L. Bian, R. Ding and B. Zhu, Two Component Higgs-Portal Dark Matter, Phys. Lett. B 728 (2014) 105 [arXiv: 1308.3851] [INSPIRE].

[17] S. Esch, M. Klasen and C.E. Yaguna, A minimal model for two-component dark matter, JHEP 09 (2014) 108 [arXiv: 1406.0617] [INSPIRE].

[18] A. Dutta Banik, M. Pandey, D. Majumdar and A. Biswas, Two component WIMP-FImP dark matter model with singlet fermion, scalar and pseudo scalar, Eur. Phys. J. C 77 (2017) 657 [arXiv: 1612 . 08621] [INSPIRE].

[19] M. Klasen, F. Lyonnet and F.S. Queiroz, NLO+NLL collider bounds, Dirac fermion and scalar dark matter in the B-L model, Eur. Phys. J. C 77 (2017) 348 [arXiv:1607.06468] [INSPIRE].

[20] S. Bhattacharya, P. Poulose and P. Ghosh, Multipartite Interacting Scalar Dark Matter in the light of updated LUX data, JCAP 04 (2017) 043 [arXiv: 1607.08461] [INSPIRE].

[21] S. Bhattacharya, P. Ghosh, T.N. Maity and T.S. Ray, Mitigating Direct Detection Bounds in Non-minimal Higgs Portal Scalar Dark Matter Models, JHEP 10 (2017) 088 [arXiv: 1706. 04699] [INSPIRE]. 
[22] J. Herrero-Garcia, A. Scaffidi, M. White and A.G. Williams, On the direct detection of multi-component dark matter: sensitivity studies and parameter estimation, JCAP 11 (2017) 021 [arXiv: 1709.01945] [INSPIRE].

[23] M. Aoki, D. Kaneko and J. Kubo, Multicomponent Dark Matter in Radiative Seesaw Models, Front. in Phys. 5 (2017) 53 [arXiv:1711.03765] [INSPIRE].

[24] N. Khan, Neutrino mass and the Higgs portal dark matter in the ESSFSM, Adv. High Energy Phys. 2018 (2018) 4809682 [arXiv: 1707.07300] [INSPIRE].

[25] A. Ahmed, M. Duch, B. Grzadkowski and M. Iglicki, Multi-Component Dark Matter: the vector and fermion case, Eur. Phys. J. C 78 (2018) 905 [arXiv:1710.01853] [INSPIRE].

[26] J. Herrero-Garcia, A. Scaffidi, M. White and A.G. Williams, Time-dependent rate of multicomponent dark matter: Reproducing the DAMA/LIBRA phase-2 results, Phys. Rev. D 98 (2018) 123007 [arXiv:1804.08437] [INSPIRE].

[27] M. Aoki and T. Toma, Boosted Self-interacting Dark Matter in a Multi-component Dark Matter Model, JCAP 10 (2018) 020 [arXiv: 1806.09154] [INSPIRE].

[28] S. Yaser Ayazi and A. Mohamadnejad, Scale-Invariant Two Component Dark Matter, arXiv: 1808.08706 [INSPIRE].

[29] A. Poulin and S. Godfrey, Multi-component dark matter from a hidden gauged SU(3), arXiv: 1808.04901 [INSPIRE].

[30] S. Chakraborti and P. Poulose, Interplay of Scalar and Fermionic Components in a Multi-component Dark Matter Scenario, arXiv:1808.01979 [INSPIRE].

[31] J.A. Casas, D.G. Cerdeño, J.M. Moreno and J. Quilis, Reopening the Higgs portal for single scalar dark matter, JHEP 05 (2017) 036 [arXiv: 1701.08134] [INSPIRE].

[32] J. McDonald, Gauge singlet scalars as cold dark matter, Phys. Rev. D 50 (1994) 3637 [hep-ph/0702143] [INSPIRE].

[33] P. Ghosh, A.K. Saha and A. Sil, Study of Electroweak Vacuum Stability from Extended Higgs Portal of Dark Matter and Neutrinos, Phys. Rev. D 97 (2018) 075034 [arXiv:1706.04931] [INSPIRE].

[34] S. Bhattacharya, N. Sahoo and N. Sahu, Minimal vectorlike leptonic dark matter and signatures at the LHC, Phys. Rev. D 93 (2016) 115040 [arXiv:1510.02760] [INSPIRE].

[35] S. Bhattacharya, N. Sahoo and N. Sahu, Singlet-Doublet Fermionic Dark Matter, Neutrino Mass and Collider Signatures, Phys. Rev. D 96 (2017) 035010 [arXiv:1704.03417] [INSPIRE].

[36] M. Hoferichter, P. Klos, J. Menéndez and A. Schwenk, Improved limits for Higgs-portal dark matter from LHC searches, Phys. Rev. Lett. 119 (2017) 181803 [arXiv:1708.02245] [INSPIRE].

[37] L. Feng, S. Profumo and L. Ubaldi, Closing in on singlet scalar dark matter: LUX, invisible Higgs decays and gamma-ray lines, JHEP 03 (2015) 045 [arXiv: 1412.1105] [INSPIRE].

[38] S. Bhattacharya, B. Karmakar, N. Sahu and A. Sil, Flavor origin of dark matter and its relation with leptonic nonzero $\theta_{13}$ and Dirac CP phase $\delta, J H E P 05$ (2017) 068 [arXiv: 1611.07419] [INSPIRE].

[39] P. Pal, An Introductory Course of Particle Physics, Taylor \& Francis, (2014).

[40] F. Pisano and A.T. Tran, Anomaly cancellation in a class of chiral flavor gauge models, in 14 th Brazilian Meeting on Particles and Fields Caxambu, Brazil, September 29 - October 3, 1993. 
[41] K. Kannike, Vacuum Stability of a General Scalar Potential of a Few Fields, Eur. Phys. J. C 76 (2016) 324 [Erratum ibid. C 78 (2018) 355] [arXiv: 1603.02680] [INSPIRE].

[42] Particle Data Group collaboration, Review of Particle Physics, Phys. Rev. D 98 (2018) 030001 [INSPIRE].

[43] M. Aoki, M. Duerr, J. Kubo and H. Takano, Multi-Component Dark Matter Systems and Their Observation Prospects, Phys. Rev. D 86 (2012) 076015 [arXiv:1207.3318] [InSPIRE].

[44] G. Bélanger, F. Boudjema, A. Pukhov and A. Semenov, MicrOMEGAs4.1: two dark matter candidates, Comput. Phys. Commun. 192 (2015) 322 [arXiv: 1407.6129] [INSPIRE].

[45] H. Han, J.M. Yang, Y. Zhang and S. Zheng, Collider Signatures of Higgs-portal Scalar Dark Matter, Phys. Lett. B 756 (2016) 109 [arXiv:1601.06232] [INSPIRE].

[46] S. Bahrami, M. Frank, D.K. Ghosh, N. Ghosh and I. Saha, Dark matter and collider studies in the left-right symmetric model with vectorlike leptons, Phys. Rev. D 95 (2017) 095024 [arXiv: 1612.06334] [INSPIRE].

[47] B. Barman, S. Bhattacharya and M. Zakeri, Multipartite Dark Matter in $\mathrm{SU}(2)_{N}$ extension of Standard Model and signatures at the LHC, JCAP 09 (2018) 023 [arXiv:1806.01129] [INSPIRE].

[48] A. Alloul, N.D. Christensen, C. Degrande, C. Duhr and B. Fuks, FeynRules $2.0-A$ complete toolbox for tree-level phenomenology, Comput. Phys. Commun. 185 (2014) 2250 [arXiv:1310.1921] [INSPIRE].

[49] J. Alwall, M. Herquet, F. Maltoni, O. Mattelaer and T. Stelzer, MadGraph 5: Going Beyond, JHEP 06 (2011) 128 [arXiv:1106.0522] [INSPIRE].

[50] T. Sjöstrand, S. Mrenna and P.Z. Skands, PYTHIA 6.4 Physics and Manual, JHEP 05 (2006) 026 [hep-ph/0603175] [INSPIRE].

[51] J. Alwall et al., The automated computation of tree-level and next-to-leading order differential cross sections and their matching to parton shower simulations, JHEP 07 (2014) 079 [arXiv: 1405.0301] [INSPIRE].

[52] R. Placakyte, Parton Distribution Functions, in Proceedings, 31st International Conference on Physics in collisions (PIC 2011): Vancouver, Canada, August 28 - September 1, 2011, arXiv:1111.5452 [INSPIRE].

[53] J. Martin, C. Ringeval and V. Vennin, Encyclopadia Inflationaris, Phys. Dark Univ. 5-6 (2014) 75 [arXiv: 1303.3787] [INSPIRE].

[54] J. Martin, The Observational Status of Cosmic Inflation after Planck, Astrophys. Space Sci. Proc. 45 (2016) 41 [arXiv:1502.05733] [INSPIRE].

[55] K. Freese, E.I. Sfakianakis, P. Stengel and L. Visinelli, The Higgs Boson can delay Reheating after Inflation, JCAP 05 (2018) 067 [arXiv:1712.03791] [INSPIRE].

[56] R. Rangarajan and N. Sahu, Perturbative Reheating and Gravitino Production in Inflationary Models, Phys. Rev. D 79 (2009) 103534 [arXiv:0811.1866] [InSPIRE].

[57] K. Griest and D. Seckel, Three exceptions in the calculation of relic abundances, Phys. Rev. D 43 (1991) 3191 [INSPIRE]. 